\title{
Functionalized liposomes and phytosomes loading Annona muricata L. aqueous extract: Potential nanoshuttles for brain-delivery of phenolic compounds
}

\author{
Simona Mancini ${ }^{\mathrm{a}, \mathrm{b}}$, Luca Nardo ${ }^{\mathrm{a}, \mathrm{b}}$, Maria Gregori ${ }^{\mathrm{a}, \mathrm{b}}$, Inês Ribeiro ${ }^{\mathrm{c}}$, Francesco Mantegazza ${ }^{\mathrm{a}, \mathrm{b}}$, \\ Cristina Delerue-Matos ${ }^{\mathrm{c}}$, Massimo Masserini ${ }^{\mathrm{a}, \mathrm{b}}$, Clara Grosso ${ }^{\mathrm{c}, *}$ \\ ${ }^{a}$ School of Medicine and Surgery, University of Milano-Bicocca, 20900 Monza, Italy \\ b Nanomedicine Center NANOMIB, University of Milano-Bicocca, 20126 Milano, Italy

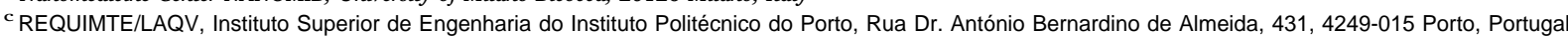

Keywords:

Annona muricata L.

Phytosomes

Liposomes

mApoE

hMAO-A

Hydrogen peroxide scavenging

\begin{abstract}
A B S T R A C T
Background: Multi-target drugs have gained significant recognition for the treatment of multifactorial diseases such as depression. Under a screening study of multi-potent medicinal plants with claimed antidepressant-like activity, the phenolic-rich Annona muricata aqueous extract (AE) emerged as a moderate monoamine oxidase A (hMAO-A) inhibitor and a strong hydrogen peroxide $\left(\mathrm{H}_{2} \mathrm{O}_{2}\right)$ scavenger.

Purpose: In order to protect this extract from gastrointestinal biotransformation and to improve its permeability across the blood-brain barrier (BBB), four phospholipid nanoformulations of liposomes and phytosomes functionalized with a peptide ligand promoting BBB crossing were produced.

Methods: AE and nanoformulations were characterized by HPLC-DAD-ESI-MS ${ }^{n}$, HPLC-DAD, spectrophotometric, fluorescence and dynamic light scattering methods. Cytotoxicity and permeability studies were carried out using an in vitro transwell model of the BBB, composed of immortalized human microvascular endothelial cells ( $h$ CMEC/D3), and in vitro $h$ MAO-A inhibition and $\mathrm{H}_{2} \mathrm{O}_{2}$ scavenging activities were performed with all samples. Results: The encapsulation/binding of $\mathrm{AE}$ was more efficient with phytosomes, while liposomes were more stable, displaying a slower extract release over time. In general, phytosomes were less toxic than liposomes in $h \mathrm{CMEC} / \mathrm{D} 3$ cells and, when present, cholesterol improved the permeability across the cell monolayer of all tested nanoformulations. All nanoformulations conserved the antioxidant potential of AE, while phosphatidylcholine interfered with MAO-A inhibition assay.

Conclusions: Overall, phytosome formulations registered the best performance in terms of binding efficiency, enzyme inhibition and scavenging activity, thus representing a promising multipotent phenolic-rich nanoshuttle for future in vivo depression treatment.
\end{abstract}

\section{Introduction}

Major depressive disorder (MDD) is a highly prevalent multi-factorial mental disorder. Since current therapies - tricyclic antidepressants (TCAs), selective serotonin and/or noradrenaline reuptake inhibitors (SSRIs, NRIs, SNRIs), and monoamine oxidase-A inhibitors (MAO-AIs) - have delayed onset of action and a substantial fraction of patients fail to respond to them (Carvalho et al., 2017), the development of new, fast-acting multi-targeted antidepressants with strong efficacy is deemed urgent to increase the range of options for patients'

\footnotetext{
Abbreviations: AE, aqueous extract; ApoE, apolipoprotein E; BBB, blood-brain barrier; CNS, central nervous system; DCM, dichloromethane; DMSO, dimethylsulfoxide; DSPE-PEG-

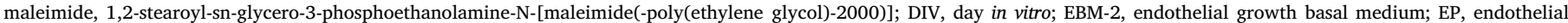

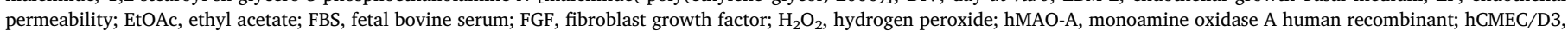

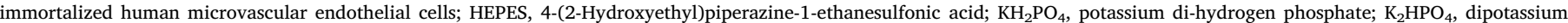

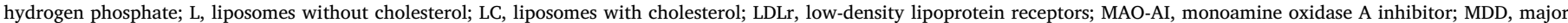

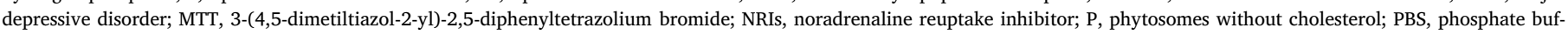

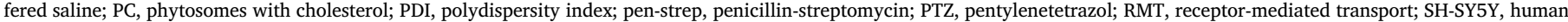

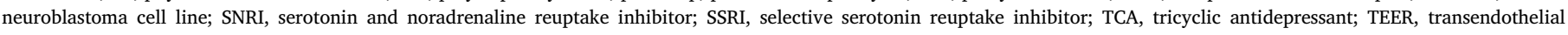
electrical resistance
} 
treatment. Thus, multi-component herbal drugs that are able to interact with several targets involved in the pathogenesis of depression are a promising choice (Yan et al., 2016).

The greatest obstacle in delivering a neuroactive agent to its target is the blood-brain barrier (BBB), a physical barrier that regulates the flow of drugs from the blood circulation to brain interstitial fluid, as well as ensures the homeostasis of the central nervous system (CNS). Its neurovascular unit is a complex system that encompasses endothelial cells, astroglia, pericytes, perivascular mast cells, and all the related interactions (Masserini, 2013). The obstruction of the paracellular pathway through tight junctions that connect the BBB's endothelial cells protects the brain from toxic molecules, while vital molecules are transcellularly transported from the blood to the brain by means of lipid-mediated free diffusion or carrier- or receptor-mediated transport (RMT) through the BBB (Re et al., 2011; Masserini, 2013; Zhou et al., 2014b). Among the several nanocarriers that have been designed to increase neuroprotective drug bioavailability, liposomes and phytosomes have attracted increasing attention. They combine the ability to incorporate and deliver large amounts of loaded compounds, with the possibility of functionalizing their surface with different ligands, such as a modified peptide derived from the receptor-binding domain of apolipoprotein E (mApoE) (Masserini, 2013). mApoE-functionalized nanoparticles can mimic lipoprotein particles that are endocytosed into the BBB endothelium via low-density lipoprotein receptors (LDLr) and can be transported across the BBB both in vitro and in vivo (Re et al., 2011; Balducci et al., 2014; Bana et al., 2014). Functionalization with mApoE has proved to be a successful approach to brain targeting. One of its breakthroughs is, for example, the production of liposomes bifunctionalized with phosphatidic acid and/or cardiolipin and with ApoE or mApoE for in vivo reduction of amyloid beta plaques, an innovation described in patent US 8877236 B2 (Masserini et al., 2014).

Polar herbal compounds, such as phenolics, are strongly active in in vitro systems but poorly absorbed in vivo due to several factors, namely their large molecular size, degradation in the gastrointestinal tract and poor lipid solubility, which limits their bioavailability (Bernardo et al., 2017). In liposomes, polar plant extracts are entrapped in the water compartment and surrounded by the phospholipid bilayer; on the contrary, in phytosomes a complex involving chemical bonds is formed between the polar substances and the polar heads of the phospholipid bilayer. This dispositional difference results in a more efficient storage of the plant extract in phytosomes than in liposomes (Ajazuddin et al., 2010; Yu et al., 2016).

In a previous paper, we described the in vitro inhibition of an aqueous extract from the leaves of Annona muricata L. over monoamine oxidase A (MAO-A) activity (Grosso et al., 2015). Moreover, the ethanolic extract has recently demonstrated its antidepressant-like effect by reducing the immobility time and increasing the swimming time in the forced swim test using Sprague-Dawley rats (Bikomo et al., 2017). In the current paper, in order to enhance the extract's ability to cross the $\mathrm{BBB}$, four different formulations of liposomes and phytosomes functionalized with $\mathrm{mApoE}$ were prepared and their cytotoxicity and permeability were assessed using the monoculture BBB model composed of immortalized human microvascular endothelial cells ( $h$ CMEC/D3). The encapsulation/binding efficiency of the extract and respective release rates for the produced formulations were assessed, as well as the conservation of the extract activity against MAO-A and as $\mathrm{H}_{2} \mathrm{O}_{2}$ scavenger upon incorporation in the drug carriers.

\section{Material and methods}

\section{Plant material}

A. muricata leaves (lots nr. 3611.184.14, 3611.241.13, 3611.028.16, 3611.252.16) were purchased from Morais e Costa \& CA. Lda (Porto, Portugal). A. muricata fruit (lot nr.1016E16) was acquired from Fiel Lourenço Lda (S. Julião do Tojal, Portugal). Seeds were separated from the rest of the fruit and desiccated at $50{ }^{\circ} \mathrm{C}$ for $48 \mathrm{~h}$. The plant material was powdered to a mean particle size below $910 \mu \mathrm{m}$ before extractions and stored at room temperature in plastic flasks protected from light.

\section{Reagents and standards}

Cholesterol and 1,2-stearoyl-sn-glycero-3-phosphoethanolamine-N[maleimide(-poly(ethylene glycol)-2000)] (DSPE-PEG-maleimide) were purchased from Avanti Polar Lipids (USA). L- $\alpha$-phosphatidylcholine from egg yolk, Type XI-E was purchased from Sigma-Aldrich (Steinheim, Germany). mApoE (CWG-LRKLRKRLLR-NH2) was purchased from DBA Italia (Segrate, Italy). The $h$ CMEC/D3 cell line was obtained under license from Institut National de la Sante et de la Recherche Medicale (INSERM, Paris, France).. Rat type I collagen, 1/ 100 chemically defined lipid concentrated, and all the media and supplements for cell culture were purchased from Gibco (Invitrogen, Paisley, UK). Monoamine oxidase A (MAO-A, human recombinant), kynuramine, tyramine, 4-aminoantipyrine, clorgyline, ascorbic acid, $p$ coumaric acid, epicatechin, 3-(4,5-dimetiltiazol-2-yl)-2,5-diphenyltetrazolium bromide (MTT), sepharose $\mathrm{Cl}-4 \mathrm{~B}$, diethyl ether, tetrahydrofuran, methanol, chloroform, dichloromethane and $\mathrm{H}_{2} \mathrm{O}_{2} 30 \% \mathrm{wt}$ were purchased from Sigma-Aldrich (St. Louis, MO, USA, and Steinheim, Germany). 5-O-Caffeoylquinic acid, quercetin-3-O-galactoside, quercetin-3-O-glucoside, quercetin-3-O-rutinoside, kaempferol-3$O$-rutinoside and catechin were obtained from Extrasynthèse (Genay, France). Formic acid, acetonitrile hipersolv, methanol hipersolv, hexane, $\mathrm{HCl} 37 \%$ and ammonium 25\% were from VWR (Fontenay-sousBois, France). Acetonitrile LC-MS grade and methanol LC-MS grade were supplied by Scharlau (Barcelona, Spain). Formic acid 98\% PA-ACS was obtained from Panreac (Barcelona, Spain). Ethyl acetate and ethanol absolute were from Carlo Erba (Val de Reuil Cedex, France). Potassium di-hydrogen phosphate $\left(\mathrm{KH}_{2} \mathrm{PO}_{4}\right)$ and dipotassium hydrogen phosphate $\left(\mathrm{K}_{2} \mathrm{HPO}_{4}\right)$ were obtained from Merck (Darmstadt, Germany) and vanillic acid from Fluka Chemie GmbH (Buchs, Switzerland).

\section{Extraction procedures}

The aqueous extract (AE) from $A$. muricata leaves was prepared by mixing $3 \mathrm{~g}$ of plant material with $500 \mathrm{ml}$ of water at $100{ }^{\circ} \mathrm{C}$ for $20 \mathrm{~min}$. After filtration, the extract was lyophilized.

The seed oil was removed using stirring maceration with hexane for $3 \mathrm{~h}$. Then, ethyl acetate (EtOAc) and dichloromethane (DCM) extracts were prepared in a proportion of $1 \mathrm{~g}$ seeds: $100 \mathrm{ml}$ of solvent according to the following steps: $30 \mathrm{~min}$ of sonication; $2 \mathrm{~h}$ of stirring maceration $(200 \mathrm{rpm})$ at room temperature; $30 \mathrm{~min}$ of sonication. Afterwards, the organic extracts were filtrated, and the solvent evaporated under reduced pressure. The obtained extraction yields were: $18.0 \pm 2.0 \%$ (leaves, AE), $3.8 \pm 0.4 \%$ (seeds, EtOAc) and $3.2 \pm 0.5 \%$ (seeds, DCM).

\section{HPLC-DAD-ESI-MS ${ }^{n}$ analyses}

Samples were injected on an HPLC Accela (Thermo Fischer Scientific, Bremen, Germany) using a C18 Spherisorb ODS2 $(25.0 \times 0.46 \mathrm{~cm} ; 5 \mu \mathrm{m}$ particle size) column from Waters (Ireland). Phenolic compounds and alkaloids were analyzed in the negative and positive ion modes, respectively. The solvent system consisted in formic acid $1 \%$ (A) and methanol (B), starting with $5 \%$ B. The gradient installed was according to Grosso et al., (2015) with a solvent flow rate of $0.92 \mathrm{ml} / \mathrm{min}$. Acetogenins were analyzed in the positive ion mode according to the gradient proposed by Le Ven et al. (2014): $40 \%$ B at $0 \mathrm{~min}, 100 \% \mathrm{~B}$ at $25 \mathrm{~min}$, and held for $15 \mathrm{~min}$ at $100 \% \mathrm{~B}$. Two eluent systems were tested, water $(A)$ and acetonitrile $(B)$ and formic acid $1 \%$ (A) and acetonitrile (B), the last one giving higher intensities in MS. Chromatograms were recorded at 220, 280, 320 and $350 \mathrm{~nm}$ in order to explore the presence of acetogenins, alkaloids and phenolic compounds. 
Analyses were performed on an LTQ Orbitrap ${ }^{\mathrm{TM}}$ XL hybrid mass spectrometer (Thermo Fischer Scientific, Bremen, Germany) controlled by LTQ Tune Plus 2.5.5 and Xcalibur 2.1.0, in negative and positive mode. The capillary voltage of the electrospray ionization source (ESI) was set to $3.0 \mathrm{kV}$ (negative mode) and $3.1 \mathrm{kV}$ (positive mode). The capillary temperature was $275^{\circ} \mathrm{C}$. The sheath gas and auxiliary gas flow rate were at 40 and 10 (arbitrary unit as provided by the software settings). The capillary voltage was $-35 \mathrm{~V}$ (negative mode) or $48 \mathrm{~V}$ (positive mode) and the tube lens voltage $-200 \mathrm{~V}$ for negative mode and $120 \mathrm{~V}$ for positive mode.

MS data handling software (Xcalibur QualBrowser software, Thermo Fischer Scientific) was used to search for predicted metabolites by their $m / z$ value. All peaks were check for $m / z$ value and fragmentation products.

Compounds were identified by comparing their retention times, MS and UV-vis spectra with pure standards and data from literature.

\section{HPLC-DAD analyses}

AE was analyzed on an analytical HPLC unit (Shimadzu) as previously described (Moreira et al., 2017) using the same gradient systems as for HPLC-DAD-ESI-MS ${ }^{n}$ analyses. The eluent systems consisted in formic acid 5\% (A) and methanol (B) (for phenolic compounds and alkaloids) or water (A) and acetonitrile (B) (for acetogenins).

The quantification of phenolic compounds was achieved using external calibration curves obtained at $280 \mathrm{~nm}$ (for flavan-3-ols and procyanidins), $320 \mathrm{~nm}$ (for hydroxycinnamic acids) and $350 \mathrm{~nm}$ (for flavonols) (Supplementary data, section S1, Table S1). Compounds 1-3, 5 and 7 were quantified as catechin, compound 4 as 5-O-caffeoylquinic acid, compound 6 as epicatechin, compound 8 as p-coumaric acid, compound 13 as quercetin-3-O-galactoside, compounds 14 and 17 as quercetin-3-O-glucoside, compounds $9-12,15,16,20$ and 21 as quercetin-3-O-rutinoside, and compounds $18+11$ and 22 as kaempferol-3$O$-rutinoside.

\section{Liposomes preparation}

Liposomes with two different compositions were prepared. One, by mixing L- $\alpha$-phosphatidylcholine/cholesterol/DSPE-PEG-maleimide (65:32.5:2.5, M:M:M), and the other, composed by $97.5 \mathrm{~mol} \% \mathrm{~L}-\alpha-$ phosphatidylcholine and 2.5 mol\% DSPE-PEG-maleimide. Lipids were dissolved in chloroform. The solvent was evaporated under reduced pressure and the lipid films were stored overnight in the freezer. Lipid films were hydrated with $100 \mu \mathrm{L}$ of borate buffer (pH 10). Four mg of the AE dissolved in $150 \mu \mathrm{L}$ of the same buffer were added to each lipid film. After 3 cycles of sequential vortexing and sonication ( $1 \mathrm{~min}$ each), 5 cycles of freezing (dry ice, $5 \mathrm{~min}$ ) - thawing $\left(65{ }^{\circ} \mathrm{C}, 5 \mathrm{~min}\right)$ and sonication $(5 \mathrm{~min})$ were performed. Subsequently, the volume was adjusted to $1 \mathrm{ml}$ with PBS and submitted to $15 \mathrm{~min}$ of additional sonication. Liposomes were gel filtered on a sepharose Cl-4B column, eluted with PBS, and the fractions containing the liposomes were collected. Further surface functionalization with mApoE peptide was carried out for BBB targeting. mApoE peptide, carrying the amino acid sequence CWG-LRKLRKRLLR and containing residues 141-150 of the receptorbinding protein of human ApoE, was reacted at room temperature overnight to form a covalent thioether bond with DSPE-PEG-maleimide, resulting in formation of liposomes functionalized with $1.25 \mathrm{~mol} \%$ mApoE. Two nanocarriers were produced by adopting this procedure: liposomes with and without cholesterol incorporating the AE (mApoELC-AE and $m A$ poE-L-AE, respectively). Non-functionalized nanocarriers (LC-AE and L-AE) were also produced for the transwell assay.

\section{Phytosomes preparation}

Three ml of tetrahydrofuran were added to a mixture containing 4:1 of phosphatidylcholine and plant extract and the reaction was stirred at
$75{ }^{\circ} \mathrm{C}$ for $4 \mathrm{~h}$ to achieve maximum yield of extract binding. After drying under reduced pressure, the mixture was diluted in chloroform and filtered through $0.45 \mu \mathrm{m}$ PTFE membranes to remove the unbound extract. The extract-phospholipid complex was further modified by adding $32.5 \mathrm{~mol} \%$ cholesterol and $2.5 \mathrm{~mol} \%$ DSPE-PEG-maleimide or just $2.5 \mathrm{~mol} \%$ DSPE-PEG-maleimide. The lipid film was rehydrated with PBS and submitted to three cycles of vortexing/sonication $(1+1 \mathrm{~min})$ and additional sonication for $15 \mathrm{~min}$. Following the latter procedure, both types of phytosomes were functionalized with $1.25 \%$ of $\mathrm{mApoE}$ by overnight incubation. Two phytosome formulations were produced using this methodology: phytosomes with and without cholesterol complexed with the AE (mApoE-PC-AE and $\mathrm{mApoE}-\mathrm{P}-\mathrm{AE}$, respectively). Non-functionalized phytosomes (PC-AE and P-AE) were also produced for the transwell assay.

\section{Characterization of liposomes and phytosomes}

The size and polydispersity index (PDI) of diluted mApoE-L-AE, mApoE-LC-AE, mApoE-P-AE and mApoE-PC-AE were measured at $25^{\circ} \mathrm{C}$ by dynamic light scattering (DLS) with a Z90-Plus device (Brookhaven Instruments Corporation, Holtsville, NY, U.S.A.). The amount of phosphatidylcholine/cholesterol was quantified by the Stewart's method (ammonium ferrothiocyanate) at $485 \mathrm{~nm}$ (Stewart, 1980).

In order to determine the amount of $\mathrm{AE}$ incorporated within the liposomes and phytosomes, an aliquot of the nanoformulation solution was withdrawn after decoration with $\mathrm{mApoE}$, processed with 2:1 chloroform: methanol so as to disrupt the drug carrier and quantified by measuring the absorbance at $272 \mathrm{~nm}$ with a Jasco 530 spectrophotometer, since the extract presents the main absorption maximum at this wavelength. Empty nanocarriers processed with 2:1 chloroform: methanol were used as the baseline. Moreover, each nanoformulation was submitted to HPLC-DAD analysis, using the same procedures adopted for the extracts.

As an independent assessment of the quantity of AE, the intensity of the fluorescence emitted at $469 \mathrm{~nm}$ by the same solution upon excitation at $393 \mathrm{~nm}$ was also measured with a Cary Eclipse spectrofluorimeter and compared to a calibration curve obtained for the extract dissolved in milliQ water (see Supplementary data, section S3, Fig. S3).

The leakage over time was assessed by means of the same methods, keeping the formulations under dialysis for as long as 4 days and withdrawing sample aliquots for separation and quantification of the extract at different times.

The maintenance of $\mathrm{pH}=10$ within the mApoE-L-AE and mApoELC-AE was assessed over time by fluorescence spectroscopy as detailed in the Supplementary data.

\section{Cell cultures}

hCMEC/D3 cells were used as a representative human BBB model. They were cultured at $37{ }^{\circ} \mathrm{C}, 5 \% \mathrm{CO}_{2} /$ saturated humidity in EBM-2 medium supplemented with $5 \% \mathrm{FBS}, 1 \%$ pen-strep, $1.4 \mu \mathrm{M}$ hydrocortisone, $5 \mu \mathrm{g} / \mathrm{ml}$ ascorbic acid, 1/100 chemically defined lipid concentrate, $10 \mathrm{mM}$ HEPES and $1 \mathrm{ng} / \mathrm{ml}$ basic FGF (Bana et al., 2014; Mancini et al., 2016). For the MTT assays, cells (passage 25-35) were seeded in a collagen coated 96 well-plate at a density of 10,000 cells/ well.

\section{MTT assay}

Cells were treated with different doses of AE, mApoE-L-AE, mApoELC-AE, mApoE-P-AE and mApoE-PC-AE for $3 \mathrm{~h}$ and the cell viability was then evaluated by means of the MTT assay (Re et al., 2011). MTT was dissolved in PBS $(0.5 \% \mathrm{w} / \mathrm{v})$, added to the cells and incubated for $2 \mathrm{~h}$ at $37^{\circ} \mathrm{C}$ in a humidified chamber. After incubation, ethanol was added to each well to dissolve the formazan crystals formed. 
Absorbance at $550 \mathrm{~nm}$ was measured using a microplate reader (Victor3 1420 Multilabel Counter, Perkin Elmer, Boston, MA, USA). Untreated cells were used as a negative control.

\section{Transwell assay}

In order to set up the BBB model for permeability experiments, $h \mathrm{CMEC} / \mathrm{D} 3$ cells (passage 25 - 35) were cultured on 12-well transwell inserts coated with type I collagen in a density of $5 \times 10^{4} \mathrm{cells} / \mathrm{cm}^{2}$. The layer of endothelial cells separates this system into an apical 'blood' side $(0.5 \mathrm{ml}$ of volume) and a basolateral 'brain' side ( $1 \mathrm{ml}$ of volume) compartment. Bioelectrical properties of the cell monolayer were checked by measuring the transendothelial electrical resistance (TEER) with STX2 electrode Epithelial Volt-Ohm meter (World Precision Instruments, Sarasota, Florida). Morphological and functional properties of the cell monolayer were evaluated by examining cells under microscope, as well as by measuring the paracellular permeability of $250 \mu \mathrm{M}$ FITC-dextran (MW $4 \mathrm{kDa}$ ) and the transcellular permeability of $76 \mathrm{nM}\left[{ }^{3} \mathrm{H}\right]$-propranolol $(0.5 \mu \mathrm{Ci})$, as previously described (Bana et al., 2014). Cell viability was also assessed by MTT assay (Orlando et al., 2013).

The cellular transport of AE, mApoE-L-AE, mApoE-LC-AE, mApoEP-AE and mApoE-PC-AE was estimated by placing $0.5 \mathrm{ml}$ of samples $(100 \mu \mathrm{g} / \mathrm{ml}$ of $\mathrm{AE})$ suspension in PBS on the upper chamber followed by incubation at $37^{\circ} \mathrm{C}$ for $3 \mathrm{~h}$. The endothelial permeability (EP) of $\mathrm{AE}$, mApoE-L-AE, mApoE-LC-AE, mApoE-P-AE and mApoE-PC-AE across the cell monolayers was estimated by measuring the absorbance of the $\mathrm{AE}$ at different times (up to $3 \mathrm{~h}$ ) in the upper and lower chambers by means of a spectrophotometer (wavelength $=272 \mathrm{~nm}$ ), and calculated as described before (Cecchelli et al., 1999). Nanocarriers without mApoE were also tested (L-AE, LC-AE, P-AE and PC-AE).

\section{MAO-A inhibition assays}

The inhibition of MAO-A activity was evaluated using two methods. One of them was based on the horseradish peroxidase-linked assay described by Holt et al. (Holt et al., 1997), with the modifications proposed in subsequent papers (Stafford et al., 2007; Grosso et al., 2013). The reaction was monitored for $20 \mathrm{~min}$ at $490 \mathrm{~nm}$ in a microplate reader Biotek Synergy HT (Winooski, Vermont, USA). The other method was based on the production of 4-hydroxyquinoline from the deamination of kynuramine (Weissbach et al., 1960) and it was applied, with minor modifications, by adopting the procedure described in ensuing papers (Dittmann et al., 2004; Novaroli et al., 2005). When employing this method, $5 \mu \mathrm{l}$ of $\mathrm{AE}$ and nanoformulations (mApoE-L-AE, mApoE-LC-AE, mApoE-P-AE and mApoE-PC-AE) were mixed with $350 \mu \mathrm{l}$ of potassium phosphate buffer $(0.1 \mathrm{M}, \mathrm{pH} 7.4)$ and $20 \mu \mathrm{l}$ of kynuramine $(3.75 \mathrm{mM})$ and the reaction was initiated with $125 \mu \mathrm{l}$ of MAO-A $(17 \mathrm{U} / \mathrm{ml})$. The production of 4-hydroxyquinoline was determined by measuring the absorbance at $314 \mathrm{~nm}$ with a Shimadzu UV260 spectrophotometer (Kyoto, Japan), after incubation at $37^{\circ} \mathrm{C}$ for $70 \mathrm{~min}$. For each tested concentration, a blank sample (without MAO-A) was used for background subtraction. mApoE-P-AE and mApoE-PC-AE were dissolved in a mixture of buffer with DMSO and, in both methods, a concentration of DMSO below 6\% did not affect the enzyme activity. The $\mathrm{IC}_{50}$ values were calculated by performing three independent assays in triplicate and comparing them with the ones obtained for clorgyline (positive control).

\section{Hydrogen peroxide scavenging activity}

The adopted procedure to evaluate the AE's scavenging ability against $\mathrm{H}_{2} \mathrm{O}_{2}$ was based on the disappearance of the $\mathrm{H}_{2} \mathrm{O}_{2}$ signal at $230 \mathrm{~nm}$ (Gülçin et al., 2006). In each tube, $425 \mu \mathrm{l}$ of AE or mApoE-L-AE, mApoE-LC-AE, mApoE-P-AE and mApoE-PC-AE dissolved in $0.1 \mathrm{M}$ phosphate buffer ( $\mathrm{pH}$ 7.4) (or merely buffer for the negative control) were mixed with $75 \mu \mathrm{l}$ of $40 \mathrm{mM}$ solution of $\mathrm{H}_{2} \mathrm{O}_{2}$. The absorbance value at $230 \mathrm{~nm}$ was read after $10 \mathrm{~min}$ of incubation by means of a Shimadzu UV-260 spectrophotometer (Kyoto, Japan). For each tested dilution, a blank sample (without $\mathrm{H}_{2} \mathrm{O}_{2}$ solution) was used for background subtraction. Ascorbic acid was used as reference for all assays. Three independent assays were performed in triplicate.

\section{Statistical analysis}

All data were recorded as mean \pm SD or mean \pm SEM. The $\mathrm{IC}_{50}$ values were calculated using Graph Pad Prism Software Version 6. For the viability and permeability tests, samples were compared and grouped according to one-way analysis of variance (ANOVA), using Bonferroni as post-test (Graph Pad Prism Software Version 6). Differences at $p<0.05$ were considered statistically significant.

\section{Results and discussion}

\section{HPLC-DAD-ESI-MS ${ }^{n}$ and HPLC-DAD analyses}

Several phenolic compounds were detected in the aqueous extract of A. muricata. Compounds $\mathbf{1}, \mathbf{3}$ and 4 showed deprotonated molecular ions characteristic of caffeoylquinic acids $\left([\mathrm{M}-\mathrm{H}]^{-}\right.$at $\left.\mathrm{m} / \mathrm{z} 353\right)$. Base peaks at $m / z 191$ [quinic acid-H] $^{-}$(compounds 1 and 4) and at 173 [(quinic acid-H) -18$]^{-}$(compound 3) were obtained from $\mathrm{MS}^{2}$ [353] fragmentation. Taking into consideration their $R t$ and their fragmentation pattern (Clifford et al., 2003), these compounds can be assigned as 3-O-caffeoylquinic acid (1), 4-O-caffeoylquinic acid (3), and 5-Ocaffeoylquinic acid (4). All these compounds were previously described in A. muricata (Marques et al., 2009; Nawwar et al., 2012). The UV spectra of peaks 1 and 3 denote co-elution with other class of compounds, which was confirmed by MS spectra. Therefore, a fragmentation pattern characteristic of procyanidin derivatives was observed, with a deprotonated molecular ion at $m / z 577$ being fragmented into characteristic ions at $m / z 425$ (base peak, retro-Diels-Alder(RDA) cleavage), 407 [425-18] $^{-}$, and 289 [catechin-H] ${ }^{-}$in the $\mathrm{MS}^{2}$ spectrum (Rockenbach et al., 2012; Ferraz et al., 2017). This fragmentation pattern was also observed for compound 7 (Table 1, Fig. 1A). In fact, procyanidins and procyanidin derivatives were already identified in different species of Annona genus, such as A. crassiflora (Ferraz et al., 2017; Justino et al., 2017) and A. cherimola (Santos et al., 2016).

Compounds 2 and 6 present similar UV and MS spectra $\left([\mathrm{M}-\mathrm{H}]^{-}\right.$at $\mathrm{m} / \mathrm{z} 289$ ) and their identification was confirmed with pure standards of catechin (2) and epicatechin (6). Both compounds showed characteristic $\mathrm{MS}^{2}$ fragments at $\mathrm{m} / \mathrm{z} 245$ (base peak, $\left[(\mathrm{M}-\mathrm{H})-\mathrm{CO}_{2}\right]^{-}$) and 205 (cleavage of the A-ring of the flavan-3-ol) (Rockenbach et al., 2012; Ferraz et al., 2017) (Table 1, Fig. 1A). Nawwar et al., (2012) and (George et al., 2015) have already reported the presence of these flavan-3-ol in hydroalcoholic and aqueous extracts of $A$. muricata. MS fragmentation of compound $\mathbf{5}$ showed not only a caffeic acid derivative but also a catechin derivative. The $\mathrm{MS}^{2}$ fragmentation of the ion at $\mathrm{m} / \mathrm{z}$ 179 yielded a base peak at $m / z 135$ which corresponded to [(caffeic acid-H) $\left.-\mathrm{CO}_{2}\right]^{-}$. However, in the MS spectrum is also observed an ion at $m / z 353$ (which may be a precursor of $m / z$ 179), indicating that the peak does not correspond to caffeic acid but to a caffeic acid derivative. Moreover, this peak also contains a catechin derivative since a base peak at $m / z 289$ [catechin-H] ${ }^{-}$is observed in the $\mathrm{MS}^{2}$ fragmentation of the ion at $m / z 469$ (Table 1, Fig. 1A).

The chromatographic behavior of compound 8, as well as its UV and MS spectra (Rt $26.03 \mathrm{~min}$; UV $309 \mathrm{~nm}$; [M-H] ${ }^{-}$at $m / z 163, \mathrm{MS}^{2}[163]$ : $119(100 \%))$ was coincident with those of $p$-coumaric acid standard (Ferreres et al., 2013), a compound already reported in this species (George et al., 2015).

Compounds 9-17 and 19-21 correspond to glycosylated quercetin derivatives. The assignment of compounds 13 and 14 was confirmed by injection of pure standards of quercetin-3-O-galactoside (13) and 
Table 1

Phenolic composition of the aqueous extract from A. muricata leaves. Results are expressed as mean \pm SD of three analyses.

\begin{tabular}{|c|c|c|c|c|c|c|}
\hline & Compound & $R t$ (min) & $\mathrm{UV}(\mathrm{nm})$ & {$[\mathrm{M}-\mathrm{H}]^{-}, m / z$} & $\mathrm{MS}^{2}[\mathrm{M}-\mathrm{H}]^{-}, m / z(\%)$ & Amount (mg/g extract, d.w.) \\
\hline \multirow[t]{2}{*}{1} & 3-O-Caffeoylquinic acid + procyanidin derivative & 10.21 & 287,325 & 353.08833 & 191(100), 179(48) & $2.16 \pm 0.05$ \\
\hline & & & & 577.13576 & 425(100), 407(81), 289(25) & \\
\hline 2 & Catechin & 13.80 & 279 & 289.07188 & 245(100), 205(35) & $1.93 \pm 0.03$ \\
\hline \multirow[t]{2}{*}{3} & 4-O-Caffeoylquinic acid + procyanidin derivative & 16.05 & 283,326 & 353.08842 & $173(100), 179(60)$ & $4.64 \pm 0.02$ \\
\hline & & & & 577.13667 & $425(100), 407(67), 289(22)$ & \\
\hline 4 & 5-O-Caffeoylquinic acid & 17.37 & $248,293 \mathrm{sh}, 326$ & 353.08888 & 191(100), 179(4) & $4.14 \pm 0.04$ \\
\hline \multirow[t]{2}{*}{5} & Caffeic acid derivative + catechin derivative & 18.53 & 286,323 & 179.03537 & $135(100)$ & $4.70 \pm 0.55$ \\
\hline & & & & 469.11460 & $289(100)$ & \\
\hline 6 & Epicatechin & 19.85 & 279 & 289.07230 & 245(100), 205(34), 179(13) & $6.23 \pm 0.01$ \\
\hline 7 & Procyanidin derivative & 22.40 & $283,308 \mathrm{sh}$ & 577.13574 & 425(100), 407(19), 289(17) & $3.52 \pm 0.50$ \\
\hline 8 & $p$-Coumaric acid & 26.03 & 309 & 163.04045 & $119(100)$ & $2.45 \pm 0.52$ \\
\hline 9 & Quercetin-hexoside-rhamnosyl-pentoside & 31.19 & 265,345 & 741.18909 & 579(100), 462(22), 300(5) & $0.79 \pm 0.00$ \\
\hline 10 & Quercetin di-O-hexoside & 35.18 & $259,267,349$ & 625.14197 & $301(61), 300(100)$ & $0.90 \pm 0.00$ \\
\hline 11 & Quercetin di-O-hexoside & 35.86 & 265,347 & 625.14210 & $301(37), 300(100)$ & $0.86 \pm 0.01$ \\
\hline 12 & Rutin-O-pentoside & 41.04 & $257,267,354$ & 741.18977 & $609(40), 301(49), 300(100)$ & $4.11 \pm 0.01$ \\
\hline 13 & Quercetin-3-O-galactoside & 43.04 & $256,267,356$ & 463.09034 & $301(100)$ & $2.71 \pm 0.02$ \\
\hline 14 & Quercetin-3-O-glucoside & 43.40 & $257,267,355$ & 463.08977 & $301(100)$ & $1.71 \pm 0.02$ \\
\hline 15 & Quercetin-3-O-rutinoside & 43.85 & $256,267,355$ & 609.14818 & $301(100)$ & $5.11 \pm 0.01$ \\
\hline 16 & Quercetin-3-O-robinobioside & 44.34 & 257,350 & 609.14658 & $301(100)$ & $0.39 \pm 0.01$ \\
\hline 17 & Quercetin-3-O-pentoside & 45.09 & $258,267,354$ & 433.07868 & $301(75), 300(100)$ & $1.43 \pm 0.01$ \\
\hline 18 & Kaempferol-3-O-hexoside & 46.11 & 266,340 & 447.09401 & 285(57), 284(100) & $0.72 \pm 0.03(18+19)$ \\
\hline \multirow[t]{2}{*}{19} & $\begin{array}{l}\text { Quercetin-rhamnosyl-hexoside + Kaempferol-rhamnosyl- } \\
\text { hexoside }\end{array}$ & 46.43 & 266,340 & 609.14664 & 301(37), 300(100) & \\
\hline & & & & 593.15149 & 285(100) & \\
\hline 20 & Quercetin-hexosyl-rhamnoside & 46.76 & $258,267,348$ & 609.14680 & $301(29), 300(100)$ & $2.10 \pm 0.02$ \\
\hline 21 & Quercetin-pentosyl-rhamnoside & 47.06 & $256,266,348$ & 579.13807 & $301(14), 300(100)$ & $5.33 \pm 0.05$ \\
\hline \multirow[t]{2}{*}{22} & Kaempferol-3-O-rutinoside & 47.68 & 265,347 & 593.15131 & $285(100)$ & $0.50 \pm 0.00$ \\
\hline & Total & & & & & $56.43 \pm 1.36$ \\
\hline
\end{tabular}

quercetin-3-O-glucoside (14) $\left([\mathrm{M}-\mathrm{H}]^{-}\right.$at $m / z 463$ and a base peak at $m /$ z 301 in $\mathrm{MS}^{2}$, corresponding to the loss of the hexosyl radical, $=162$ amu) (Table 1, Fig. 1A) (Nawwar et al., 2012). With a deprotonated molecular ion $30 \mathrm{amu}$ less than those of compounds 13 and 14 and a base peak at $m / z 300$ in $\mathrm{MS}^{2}$ [433], compound 17 is a quercetin-3-Opentoside, probably quercetin-3-O-arabinoside (Table 1 , Fig. $1 \mathrm{~A}$ ) already found in A. crassiflora (Lage et al., 2014). MS spectra of compound 9 presented a deprotonated molecular ion at $m / z 741$, which produced fragments at $m / z 579$ ( $-162 \mathrm{amu}$, hexosyl radical), 462/463 ( -278 amu, rhamnosyl-pentosyl radical) and 299/300 (-441 amu, hexosyl-rhamnosyl-pentoside-H radical). Since ions resulting from the loss of the sugar moieties are more intense than that of the aglycone in $\mathrm{MS}^{2}$, sugar molecules are probably linked to different phenolic hydroxyl groups (Amaral et al., 2005; Ferreres et al., 2008). In the $\mathrm{MS}^{3}$ [741], a base peak at $m / z 300$ [aglycone- $2 \mathrm{H}^{-}$was observed (Amaral et al., 2005). Therefore, compound 9 can be tentatively labelled as quercetinhexoside-rhamnosyl-pentoside (Table 1, Fig. 1A). Compounds 10 and 11 possess the same MS fragmentation pattern, with a deprotonated molecular ion at $m / z 625$. The $\mathrm{MS}^{2}$ [625] fragmentation yielded fragments at $m / z 505$ [M-H-120] $^{-}$, 463[M-H-162] ${ }^{-}$(loss of hexosyl radical), 445[M-H-162-18 $]^{-}$(loss of hexosyl and water), and a base peak at 300 [aglycone- $2 \mathrm{H}]^{-}$. Thus, both compounds correspond to quercetin-di-O-hexosides (Table 1, Fig. 1A). Compound 12 can be tentatively identified as rutin- $O$-pentoside since in the MS fragmentation a deprotonated molecular ion at $m / z 741$ is observed. In the $\mathrm{MS}^{2}$ fragmentation, ions at $\mathrm{m} / \mathrm{z} 609$ [M-H-pentosyl] ${ }^{-}$and a base peak at $\mathrm{m} / \mathrm{z}$ 300 , which correspond to quercetin aglycone, were produced (Table 1 , Fig. 1A) (Ferraz et al., 2017). Compounds 15 and 16 are isomers. These two compounds possess the same deprotonated molecular ion $(\mathrm{m} / \mathrm{z}$ at 609 ) and a base peak corresponding to $301 / 300$ [aglycone- $\mathrm{H} / 2 \mathrm{H}]^{-}$was observed in the $\mathrm{MS}^{2}[\mathrm{M}-\mathrm{H}]^{-}$fragmentation. Therefore, compound 15 is quercetin-3-O-rhamnosyl-(1 $\rightarrow 6$ )-glucoside (quercetin-3-O-rutinoside) which was confirmed by injection of the pure standard and compound 16 is an isomer and can tentatively be assigned as quercetin-3-Orhamnosyl-( $1 \rightarrow 6)$-galactoside (=quercetin-3-O-robinobioside). In both, ${ }^{0,2} \mathrm{X}_{0}{ }^{-}$at $m / z 343$ was observed, confirming $1 \rightarrow 6$ interglycosidic linkage (Cuyckens et al., 2001) (Table 1, Fig. 1A). Indeed, these two compounds were already identified in this species (Matsushige et al., 2012b; Nawwar et al., 2012). In the MS fragmentation of compound 20 and 21, besides their $[\mathrm{M}-\mathrm{H}]^{-}$at $m / z 609$ and $m / z 579$, an ion at $m / z$ 447 was observed, corresponding to the loss of an hexosyl radical [(M$\mathrm{H})-162]^{-}$and pentosyl radical $[(\mathrm{M}-\mathrm{H})-132]^{-}$, respectively. Therefore, these compounds can be tentatively identified as quercetin-hexosyl-rhamnoside (20) and quercetin-pentosyl-rhamnoside (21). Since [Aglycone- $\mathrm{H} / 2 \mathrm{H}]^{-}$is the base peak in both $\mathrm{MS}^{2}$ spectra, both sugars are linked to the same phenolic carbon of the quercetin aglycone (Amaral et al., 2005; Ferreres et al., 2008) (Table 1, Fig. 1A). Peak 19 showed a fragmentation pattern characteristic of quercetin as well as kaempferol derivatives, showing a co-elution of two compounds. The $\mathrm{MS}^{2}$ fragmentation of the deprotonated molecular ion at $m / z 609$ originated ions at $m / z 463[(\mathrm{M}-\mathrm{H})-146]^{-}, 429\left[(\mathrm{M}-\mathrm{H})^{-}-(162+18)\right]^{-}$ and 300 [Aglycone-2H] ${ }^{-}$as base peak. Additionally, MS $^{2}$ [593] yielded an ion at $m / z 447[(\mathrm{M}-\mathrm{H})-146]^{-}$and a base peak at $m / z 285$. Thus, peak 19 contains a quercetin-rhamnosyl-hexoside and a kaempferolrhamnosyl-hexoside (Table 1, Fig. 1A). In both compounds, a $1 \rightarrow 6$ interglycosidic linkage was confirmed by the presence of the ions at $m / z$ 343 (for the quercetin derivative) and 327 (for the kaempferol derivative) (Cuyckens et al., 2001). Other glycosylated kaempferol derivatives were also detected. Compound 18 showed a $[\mathrm{M}-\mathrm{H}]^{-}$at $m / z 447$ and a $\mathrm{MS}^{2}$ [447] fragmentation yielding a fragment $162 \mathrm{amu}$ lower $(\mathrm{m} / \mathrm{z}$

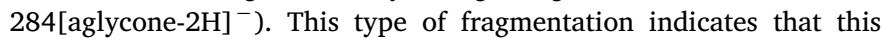
compound is a kaempferol-O-hexoside (Table 1, Fig. 1A); however, by comparison with pure standard, the compound is not kaempferol-3-Oglucoside. Finally, compound $\mathbf{2 2}$ was characterized by an MS fragmentation characteristic of kaempferol-3-O-rutinoside, already described in this species by Nawwar et al., (2012) and Matsushige et al., (2012b): $[\mathrm{M}-\mathrm{H}]^{-}$at $\mathrm{m} / \mathrm{z} 593$, another characteristic ion at $\mathrm{m} / \mathrm{z} 447$ (=146 amu, rhamnosyl radical) and, in the $\mathrm{MS}^{2}$ [593], a base peak at $m / z 285$ [kaempferol-H] ${ }^{-}$(Table 1, Fig. 1A). Compound's identity was also confirmed by comparison with a pure standard. The phenolic compounds identified accounted for $56.43 \mathrm{mg} / \mathrm{g}$ of dry extract (Table 1).

According to Matsushige et al., (2012a) and Leboeuf et al., (1981), leaves are also a source of alkaloids such as annonamine, anonaine, 

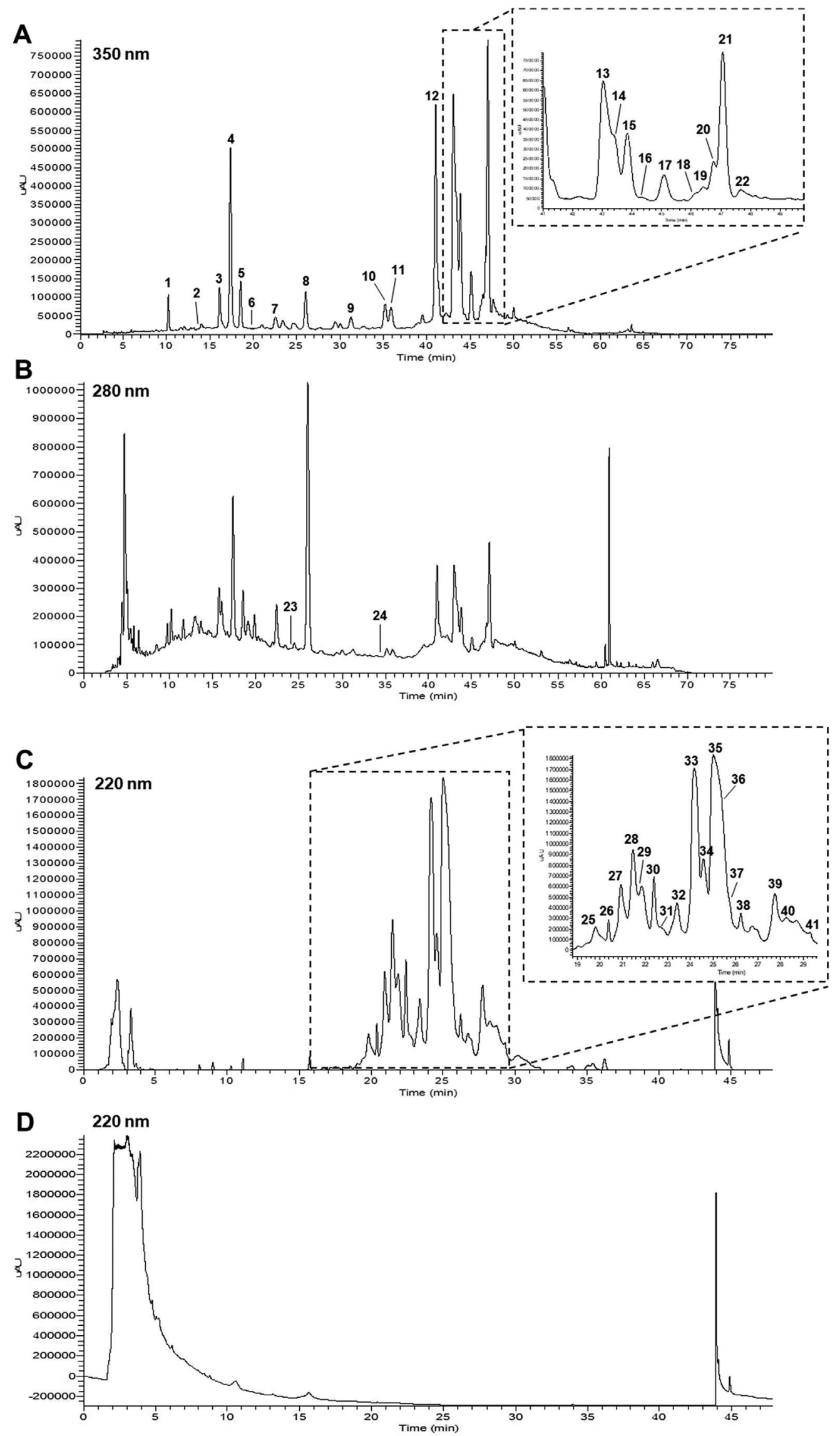

Fig. 1. HPLC-DAD chromatogram of A. muricata. (A) Detection at $350 \mathrm{~nm}$ of the aqueous extract (AE) from the leaves (negative ion mode); (B) Detection at $280 \mathrm{~nm}$ of the AE from the leaves (positive ion mode); (C) Detection at $220 \mathrm{~nm}$ of the dichloromethane extract (DCM) from the seeds (positive mode); (D) Detection at $220 \mathrm{~nm}$ of the $\mathrm{AE}$ from the leaves (positive ion mode). Identity of the compounds as in Table 1 and as described in "HPLC-DAD-ESI-MS ${ }^{n}$ and HPLC-DAD analyses" section. 

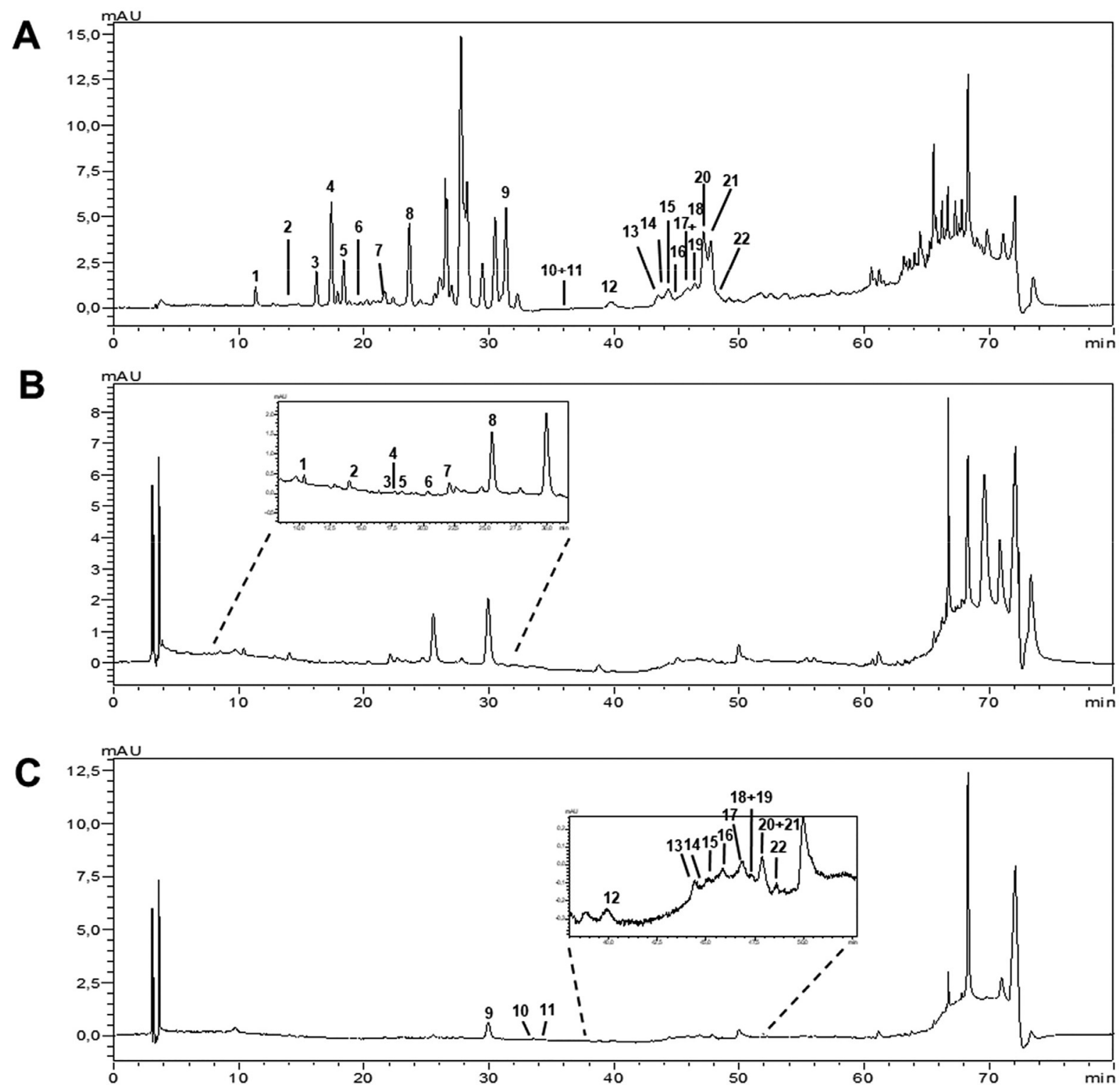

Fig. 2. HPLC-DAD chromatogram of phytosomes with cholesterol (PC-AE) (A) and liposomes with cholesterol (LC-AE) (B, C). Detection at $350 \mathrm{~nm}$ (A and C) and $320 \mathrm{~nm}(\mathrm{~B})$.

O,O-dimethylcoclaurine, 4'-O-methylcoclaurine, (+)-norcorydine, coclaurine, reticuline and stepharine. Based on MS spectra, only trace amounts of coclaurine (23, Rt: $24.37 \mathrm{~min} ;[\mathrm{M}+\mathrm{H}]^{+}, \mathrm{m} / \mathrm{z}: 286$; $\mathrm{MS}^{2}[\mathrm{M}+\mathrm{H}]^{+} m / z$ (\%): 269(100), 178(1), 175(5), 151(3), 137(3), 107(4)) and of reticuline (24, Rt: $34.14 \mathrm{~min} ;[\mathrm{M}+\mathrm{H}]^{+}, \mathrm{m} / z: 330$; $\mathrm{MS}^{2}[\mathrm{M}+\mathrm{H}]^{+} m / z(\%):$ 299(5), 192(100), 175(1), 137(1)) were detected (Stévigny et al., 2004; Schmidt et al., 2005; Rottscholl et al., 2016) (Fig. 1B, supplementary data, section S2, Fig. S1). The absence of almost all previously reported alkaloids may be related with the extraction procedure employed in the current study. Indeed, Matsushige et al., (2012a) extracted the alkaloid fraction with $\mathrm{MeOH}$ followed by partitioning with $n$-hexane, chloroform, ethyl acetate and buthanol successively.

Acetogenins are usually regarded as lipophilic neurotoxins (Caparros-Lefebvre et al., 1999; Champy et al., 2005). In order to assess the presence or absence of acetogenins in the aqueous extract, an organic extract (DCM) prepared from the seeds, a rich source of this class of compounds, was used for comparative purposes. Acetogenins possess UV spectra with a maximum at $210-220 \mathrm{~nm}$ and produce characteristic ion patterns consisting of $[\mathrm{M}+\mathrm{Na}]^{+}$and $[\mathrm{M}+\mathrm{H}]^{+}$molecular ions, as well as ions corresponding to consecutive losses of $\mathrm{H}_{2} \mathrm{O}(-18 \mathrm{amu})$ from the $[\mathrm{M}+\mathrm{H}]^{+}$ion (Alali et al., 1999). Therefore, the mass spectrum of peaks 25-30, 33, 34 and 38 in the DCM extract from the seeds (Fig. 1C) presented as major ion peak at $m / z 613$, corresponding to the $[\mathrm{M}+\mathrm{H}]^{+}$, and the fragmentation of that pseudomolecular ion yielded 5 consecutive losses of $\mathrm{H}_{2} \mathrm{O}(\mathrm{m} / z 595,577,559,541,523$ and 505) as shown in Fig. S2 (supplementary data, section S2). The mass spectrum of peaks 31, 32, 35-37, 39-41 showed a major ion peak at $m / z 597$ and, once again, 5 consecutive losses of $\mathrm{H}_{2} \mathrm{O}$, yielding the ions at $\mathrm{m} / \mathrm{z}$ 579, 591, 543, 525 and 507 (Fig. S2, supplementary data, section S2). Some acetogenins with molecular mass of 612 and $596 \mathrm{~g} / \mathrm{mol}$ are described in the literature (Alali et al., 1999). Regarding the AE, as it can be seen in the chromatogram (Fig. 1D), and analysing the UV and MS spectra, we concluded that acetogenins where not detected in this extract, which suggests reduced side effects for pharmaceutical formulations derived from this source. According to some authors (CaparrosLefebvre et al., 1999; Champy et al., 2005), over-consumption of $A$. muricata may have caused an atypical Parkinsonism on the islands of Guadeloupe and Guam due to the presence of acetogenins. On the other hand, Lannuzel et al., (2007) observed that nearly $50 \%$ of the atypical 
parkinsonism patients were not heavy consumers of annonaceaous products while some control patients consumed them in large amounts, which indicates that environmental or genetic factors might be also involved. Champy et al., (2005) demonstrated that a dose of $106 \mathrm{mg} / \mathrm{kg}$ of annonacin, when infused intravenously, induced neurodegeneration in rats, and that a daily consumption of $A$. muricata pulp, nectar, leaf infusion, or leaf decoction corresponds to the ingestion of 78, 188, 0.73 and $0.73 \mathrm{mg} / \mathrm{kg}$ of annonacin per year, respectively. These results showed that aqueous extraction from the leaves does not allow to obtain high amounts of these class of lipophilic compounds and, in fact, our extraction method did not permit to detect acetogenins by HPLCDAD-ESI-MS ${ }^{n}$. To further reinforce the safety of polar extracts from $A$. muricata leaves, Bikomo et al., (2017) and N'Gouemo et al., (1997) reported that the ethanolic extract displayed antidepressant-like effects on rats at 50, 150 and $300 \mathrm{mg} / \mathrm{kg}$ and suppressed the tonic phase of pentylenetetrazol (PTZ)-induced clonic-tonic seizures in mice at 5-100 mg/kg, respectively. Moreover, Florence et al., (2014) observed the antidiabetic effect of the AE on streptozotocin-induced diabetic rats at $100 \mathrm{mg} / \mathrm{kg}$ or $200 \mathrm{mg} / \mathrm{kg}$. In conclusion, we were able to demonstrate that the decoction prepared in this study is rich in phenolic compounds, two alkaloids were in trace amounts and no acetogenins were detected.

\section{Characterization of the extract binding efficiency and release by nanoformulations}

\section{Phytosomes}

mApoE-PC-AE and mApoE-P-AE had a mean particle size of $106 \pm 9 \mathrm{~nm}$ and $161 \pm 12 \mathrm{~nm}$, respectively. Both systems were moderately polydispersed, registering PDI values of 0.245 and 0.275 , respectively, owing to the fact that the customary extrusion procedure was avoided to minimize extract losses. The phosphatidylcholine-extract binding efficiency was $49 \pm 3 \%$. The HPLC-DAD profile confirms that both pharmacologically active components of the AE (i.e. phenolic acids and flavonoids) were efficiently incorporated, enabling the identification of the corresponding peaks (Fig. 2A). The binding reaction was monitored in real time by means of fluorescence spectroscopy, and presenting a two-step kinetics, with rapid formation of a loosely bound AE-phosphatidylcholine transient complex followed by a slower, more stable and permanent complexation. The details of these experiments are reported in Supplementary data (see Section S4, Figs. S4 and S5).

In regard to the release of the entrapped $\mathrm{AE}$, while as much as $15 \pm 3 \%$ of the AE was detached from the $\mathrm{mApoE}-\mathrm{P}-\mathrm{AE}$ within the first $30 \mathrm{~min}$, only $28 \pm 3 \%$ was released after $24 \mathrm{~h}$. For mApoE-PC-AE, $16 \pm 3 \%$ and $39 \pm 2 \%$ of the $\mathrm{AE}$ were released after $30 \mathrm{~min}$ and $24 \mathrm{~h}$, respectively. Although it is established that the incorporation of cholesterol between the phospholipid bilayer confers stability to the nanoformulation and retards drug release, this is not the case for hydrophilic drugs, such as the AE of A. muricata. In fact, cholesterol cannot protect from leakage since part of the $\mathrm{AE}$ is linked to the external polar heads of phospholipids, rather than entrapped inside an inner space as it happens with liposomes (Briuglia et al., 2015). The rapid initial leakage (15-16\%) followed by a substantial deceleration of the drug loss is more peculiar, however, and deserving of an explanation. It might be interpreted in the light of the reaction kinetics. In other words, it is conceivable that, after the four $h$ incubation of the AE with phosphatidylcholine in THF at high temperature, part of the extract is still bound to the phospholipid only by means of the readily formed but weak $\mathrm{H}$-bonding pattern. This fraction of drug is easily released as soon as the phytosomes are resuspended in $\mathrm{H}$-bonding solvent (water). Conversely, the fraction of $A E$ that has completed the binding reaction and is linked to phosphatidylcholine through the slowly-forming permanent $\mathrm{H}$-bonding pattern, takes a comparably longer time to be detached from the carrier.

\section{Liposomes}

mApoE-LC-AE and mApoE-L-AE had a mean particle size of $138 \pm 29 \mathrm{~nm}$ and $195 \pm 33 \mathrm{~nm}$, with a PDI of 0.193 and 0.261 , respectively. The entrapment efficiency of the AE demonstrated by UV-Vis absorption was $26 \pm 16 \%$ (mApoE-LC-AE) and $27 \pm 7 \%$ (mApoE-L-AE). As presented in Fig. 2B and 2C, HPLC analysis indicated that both flavonoids and phenolic acids were also efficiently incorporated in the case of liposomes.

After $30 \mathrm{~min}, 8 \pm 1 \%$ (mApoE-L-AE) and $7 \pm 2 \%$ (mApoE-LC-AE) of the $\mathrm{AE}$ had been released from the liposomes and after a 24 -h period, the obtained values were $21 \pm 2 \%$ (mApoE-L-AE) and $22 \pm 2 \%$ (mApoE-LC-AE). The same results were independently confirmed by fluorescence experiments. In this case, the presence of cholesterol seemed to have a minor effect on drug leakage.

In order to verify whether the leakage was influenced by the alteration of the liposome's internal $\mathrm{pH}$, we performed fluorescence measurements as detailed in the Supplementary data (see Supplementary data, Section S5, Fig. S6 and S7). The results suggest that the internal $\mathrm{pH}$ remained $\geq 10$ over 2 days.

\section{Viability of hCMEC/D3 and permeability of nanoformulations across a cellular BBB model}

To investigate the ability of the AE, mApoE-L-AE, mApoE-LC-AE, mApoE-P-AE and mApoE-PC-AE to cross the BBB, we assembled an in vitro $\mathrm{BBB}$ transwell model composed by a monolayer of polarized endothelial cells seeded on a porous membrane, allowing an apical compartment ('blood' side) to be physically separated from a basolateral one ('brain' side). $h \mathrm{CMEC} / \mathrm{D} 3$ cells were used as representative of the human BBB model and the transwell system was characterized by measuring its bioelectrical and functional properties. In particular, the trans-electrical endothelial resistance (TEER) was monitored during the cell monolayer's formation, presenting a gradual increase from $19.7 \pm 2.3 \Omega \mathrm{cm}^{2}$ at 1 day in vitro (DIV) to $53.8 \pm 3.5 \Omega \cdot \mathrm{cm}^{2}$ at 15 DIV. Moreover, at 15 DIV, the endothelial permeability (EP) values for $\left[{ }^{3} \mathrm{H}\right]$-propranolol and FITC-dextran (MW $4000 \mathrm{Da}$ ) were in the order of $2.3 \pm 0.1 \times 10^{-3} \mathrm{~cm} / \mathrm{min}$ and $1.02 \pm 0.04 \times 10^{-6} \mathrm{~cm} / \mathrm{min}$, respectively, which was in agreement with the values reported in the literature, showing preferentially the transcellular transport (Omidi et al., 2003; Bicker et al., 2014).

Before performing the passage experiments across the in vitro model of the BBB, the possible cytotoxicity of the AE, mApoE-L-AE, mApoELC-AE, mApoE-P-AE and mApoE-PC-AE on hCMEC/D3 was assessed by MTT, since the permeability of the $h$ CMEC/D3 monolayer substantially depends on the cells integrity. Hence, each of the above formulations was tested at three extract concentrations: 30,60 and $100 \mu \mathrm{g} / \mathrm{ml}$, after $3 \mathrm{~h}$ of incubation. As depicted in Fig. 3, the AE exhibits substantial cytotoxicity resulting in less than $60 \%$ viability even at the lowest drug concentration. However, encapsulation in either of the carriers demonstrated beneficial effects on cells health, raising the viability to over $80 \%$, even in the less favorable cases $(100 \mu \mathrm{g} / \mathrm{ml})$. At the lower tested concentration, which is nonetheless already pharmacologically relevant (vide infra), nearly $100 \%$ of viability was recorded for both carriers.

The transport of $h \mathrm{CMEC} / \mathrm{D} 3$ cells was tested at $100 \mu \mathrm{g} / \mathrm{ml}$ of extract concentration, entrapped or not within the nanoformulations, in order to facilitate the spectrophotometric detection of the AE's fraction transported across the monolayer. Although the AE was able to cross the in vitro $\mathrm{BBB}$, which may have also resulted from interference with cell integrity (since the extract alone is substantially cytotoxic), the permeability increased between 4 to 9-fold with nanoformulations functionalized with mApoE (mApoE-L-AE, mApoE-LC-AE, mApoE-P-AE and $\mathrm{mApoE}-\mathrm{PC}-\mathrm{AE}$ ). This indicates that the transcellular permeability of nanocarriers does not derive from cell unviability but from increased transport caused by functionalization with mApoE. In particular, mApoE-LC-AE and mApoE-PC-AE were nearly twice more effective in 


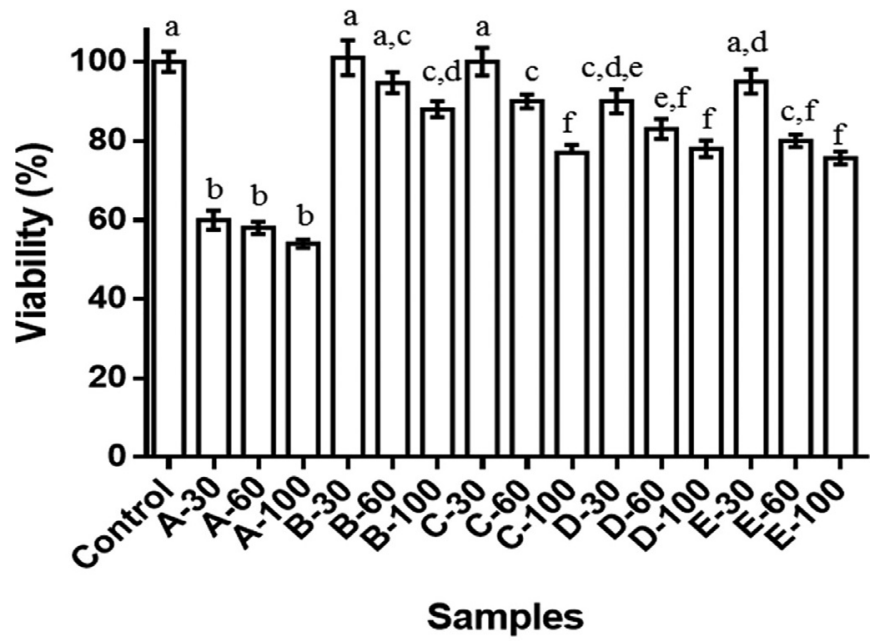

Fig. 3. Effect of the aqueous extract (AE) and nanoformulations on $h$ CMEC-D3 cells viability at the end of $3 \mathrm{~h}$. Data are presented as mean \pm SEM. Samples. A - AE; B - phytosomes functionalized with mApoE (mApoE-P-AE); C - phytosomes with cholesterol and functionalized with mApoE (mApoE-PC-AE); D liposomes functionalized with mApoE (mApoE-L-AE); E - liposomes with cholesterol and functionalized with mApoE (mApoE-LC-AE); $30-30 \mu \mathrm{g} / \mathrm{ml} ; 60$ $60 \mu \mathrm{g} / \mathrm{ml} ; 100-100 \mu \mathrm{g} / \mathrm{ml}$. Different letters mean significant differences at $p<.05$.

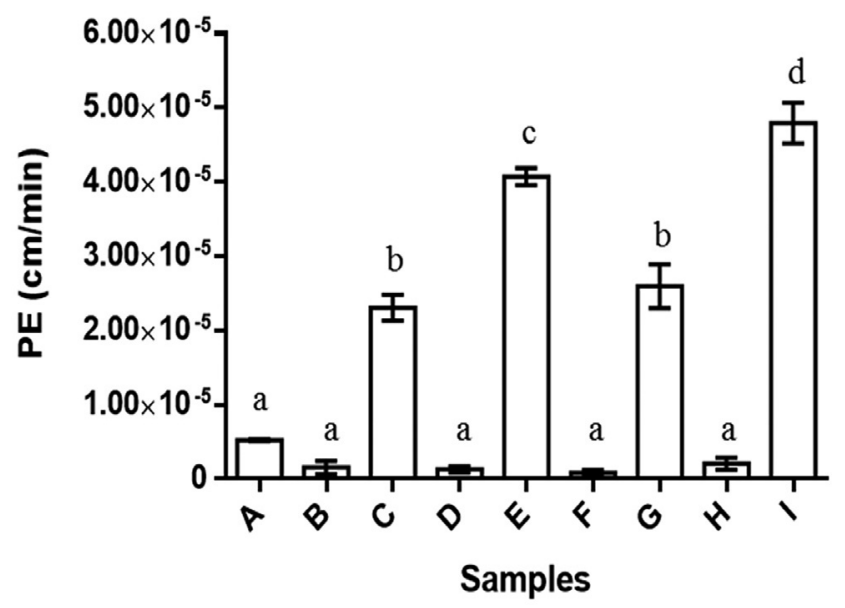

Fig. 4. Cellular uptake and transport of phytosomes, liposomes and the aqueous extract (AE) across $h \mathrm{CMEC} / \mathrm{D} 3$ monolayers. Data are presented as mean \pm SEM. A - AE; B - phytosomes (P-AE); C - phytosomes functionalized with mApoE (mApoE-P-AE); D - phytosomes with cholesterol (PC-AE); E phytosomes with cholesterol and functionalized with mApoE (mApoE-PC-AE); F - liposomes (L-AE); G - liposomes functionalized with mApoE (mApoE-L-AE); H - liposomes with cholesterol (LC-AE); I - liposomes with cholesterol and functionalized with mApoE (mApoE-LC-AE). Different letters mean significant differences at $p<.05$.

facilitating the extract transport than mApoE-L-AE and mApoE-P-AE. This outcome may possibly be related to the stability provided by cholesterol, which allows the nanoformulations to maintain a certain shape, thus enabling a better fusion with the BBB. Contrarily, without cholesterol, nanoformulations are more likely to break, crossing in less amount the BBB. Therefore, cholesterol should be included among the constituents of an optimized brain-targeted lipid-based nanocarrier. Nonetheless, the least cytotoxic nanoformulation was mApoE-P-AE ( $\approx 90 \%$ cell viability). Moreover, by comparing the permeation of mApoE-L-AE, mApoE-LC-AE, mApoE-P-AE and mApoE-PC-AE with those nanoformulations not functionalized with this peptide (L-AE, LC$\mathrm{AE}, \mathrm{P}-\mathrm{AE}$ and $\mathrm{PC}-\mathrm{AE}$ ), it became evident that this decoration plays a pivotal role in favoring the crossing of the BBB by nanoparticles (Fig. 4). Such result is already well established in the literature by several of our group's works concerning liposomes (Gobbi et al., 2010; Re et al., 2011; Bana et al., 2014) and polymeric nanocarriers (Gregori et al., 2015) as well as by other authors (Neves et al., 2016). Owing to the functionalization with mApoE peptide, transportermediated transport (namely receptor-mediated transcytosis) dominates for the nanoformulations (Re et al., 2011; Bana et al., 2014).

In vitro assessments of the extract and nanoformulations biological activities

\section{MAO-A inhibition}

Monoamine oxidases (MAOs) are flavoproteins which catalyze the oxidative deamination of several neurotransmitters, such as serotonin, noradrenaline, dopamine, tyramine, benzylamine, etc., to their corresponding aldehydes, hydrogen peroxide and ammonia (Lin et al., 2003).

In a previous work, in which the horseradish peroxidase-linked assay was used to determine the inhibition of the oxidative deamination of tyramine by MAO-A, we established that $A$. muricata AE was a good inhibitor $\left(\mathrm{IC}_{50}=19.31 \mu \mathrm{g} / \mathrm{ml}\right.$ ) (Grosso et al., 2015). In this study, so as to guarantee the reproducibility of this finding, we reevaluated a new $\mathrm{AE}$ and a similar result was obtained ( $\mathrm{IC}_{50}$ value of $12.0 \pm 0.2 \mu \mathrm{g} / \mathrm{ml}$ ). However, this peroxidase-linked assay is prone to false positive results due to different reasons: compounds with a lower oxidation-reduction potential than 4-aminoantipyrine may act as proton donors in the peroxidase reaction; phenolic substances present in the extracts may condense with the oxidized form of 4-aminoantipyrine to form colored products (Schmidt et al., 2003); and phenolic compounds with high $\mathrm{H}_{2} \mathrm{O}_{2}$ scavenging activity can deplete the concentration of this ROS, decreasing the rate of the peroxidase reaction. Bearing these in mind, in this paper the MAO-A inhibition was determined by a second method that exploits the formation of 4-hydroxyquinoline, which resulted in a notably higher $\mathrm{IC}_{50}$ value of $185.8 \pm 46.9 \mu \mathrm{g} / \mathrm{ml}$. Both $\mathrm{IC}_{50}$ values attest that the AE is pharmacologically active. Nonetheless, it was less active than the positive control, clorgyline $\left(\mathrm{IC}_{50}=25 \mathrm{ng} / \mathrm{ml}\right)$.

mApoE-L-AE, mApoE-LC-AE, mApoE-P-AE and mApoE-PC-AE were also tested to assess any loss of inhibition properties (Fig. 5). At all tested concentrations, mApoE-PC-AE was the most active nanoformulation, reaching $37.6 \%$ inhibition at $30 \mu \mathrm{g} / \mathrm{ml}$, up to $45.1 \%$ at $60 \mu \mathrm{g} / \mathrm{ml}$ (for the peroxidase-linked assay). Unfortunately, the highest concentration $(100 \mu \mathrm{g} / \mathrm{ml})$ of mApoE-P-AE and mApoE-PC-AE was not tested due to solubility problems. Nevertheless, the AE and the treated $\mathrm{AE}$ (extract submitted to the process of liposome formation), were always more active than the nanoformulations. The same pattern was observed for the assay based on the kynuramine deamination, although with a lower inhibition capacity (data not shown).

Phospholipids have been shown to affect the activity of MAO, which has a negatively charged catalytic site. In rat brain mitochondria treated with a high concentration of phospholipase A2, the activity of both isoforms, mainly of MAO-B, was able to be restored with high concentrations of the negatively charged phospholipids (phosphatidylserine, cardiolipin, and phosphatidylinositol), while the zwitterionic phospholipid phosphatydilcholine only activated MAO-A. Phosphatydilethanolamine was not active (Huang et al., 1981). In rat liver, using the same approach, similar results were achieved. MAO-B activity was restored by phosphatydilcholine, phosphatidylserine, phosphatidylinositol and phosphatydilethanolamine, with phosphatidylserine being the most effective. High amounts of phosphatydilethanolamine slightly inhibited MAO-A, whereas the other lipids increased MAO-A activity although not to the same extent as MAO-B (NavarroWelch et al., 1982). These results seem to indicate that amine substratephospholipid hydrophobic complexes may probably be formed in the presence of large amounts of phospholipid and that these complexes may be more effectively used by the mitochondrial monoamine oxidases (Navarro-Welch et al., 1982). To elucidate the mechanism by 
A

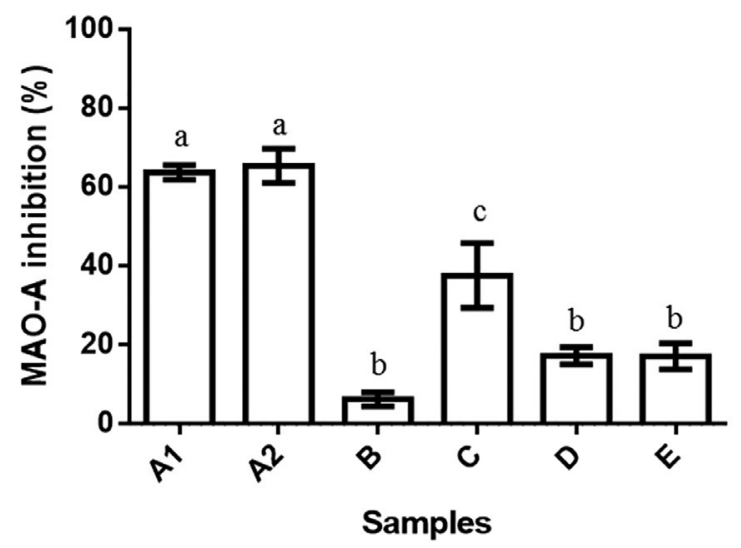

B

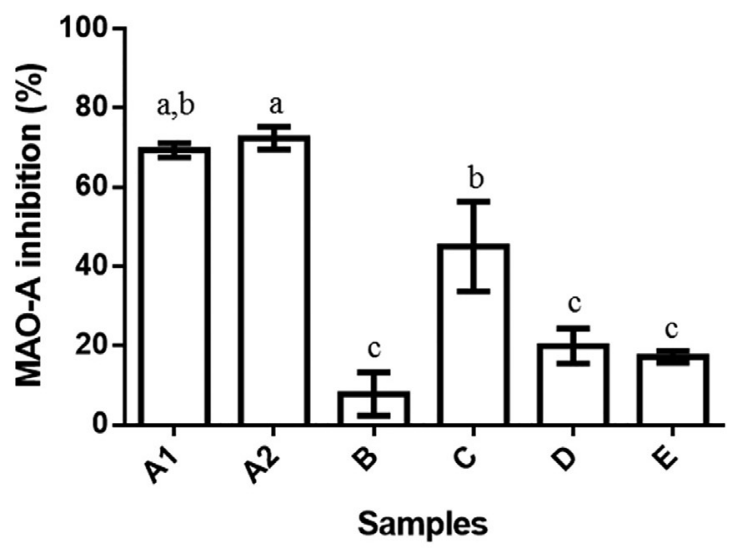

Fig. 5. MAO-A inhibition by the aqueous extract (AE) and nanoformulations assessed by the horseradish peroxidase-linked assay at $30 \mu \mathrm{g} / \mathrm{ml}$ (A) and $60 \mu \mathrm{g}$ / $\mathrm{ml}$ (B). Data are presented as mean \pm SEM. A1 - treated AE; A2 - AE; B phytosomes functionalized with mApoE (mApoE-P-AE); C- phytosomes with cholesterol and functionalized with $\mathrm{mApoE}$ (mApoE-PC-AE); D - liposomes functionalized with mApoE (mApoE-L-AE); E - liposomes with cholesterol and functionalized with mApoE (mApoE-LC-AE). Different letters mean significant differences at $p<.05$.

which negatively charged and zwitterionic phospholipids activated MAO function, Nałęcz et al. (1980) compared the $K_{m}$ value of MAO in the presence of phosphatydilcholine, phosphatydilserine, phosphatydilcholine plus phosphatidic acid and phosphatydilcholine plus tridecylamine. The lowest $K_{m}$ values were obtained for phosphatydilserine and for phosphatydilcholine plus phosphatidic acid, while the highest was achieved in the presence of positively charged amines (phosphatydilcholine + tridecylamine). In this sense, a positively charged surface would repeal the positively charged natural substrate - the biogenic amines (such as tyramine or kynuramine) - causing the concentration of substrate close to the negative charged catalytic site to decrease. Similarly, a negatively charged surface would increase the enzyme-substrate affinity, by forming the above-mentioned complexes (Reavill et al., 1978).

Based on these assumptions, it is not surprising that, in the presence of phosphatydilcholine, the extract of A. muricata was less able to inhibit MAO-A, since $K_{m}$ can be changed. Anyway, in in vivo experiments, the nanoformulations will fuse with cell membranes and release the compounds, and this limitation will be discharged.
A

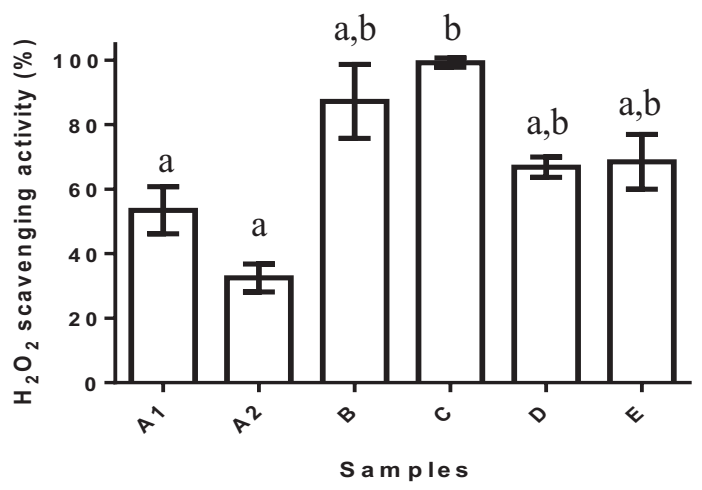

$\mathbf{B}$

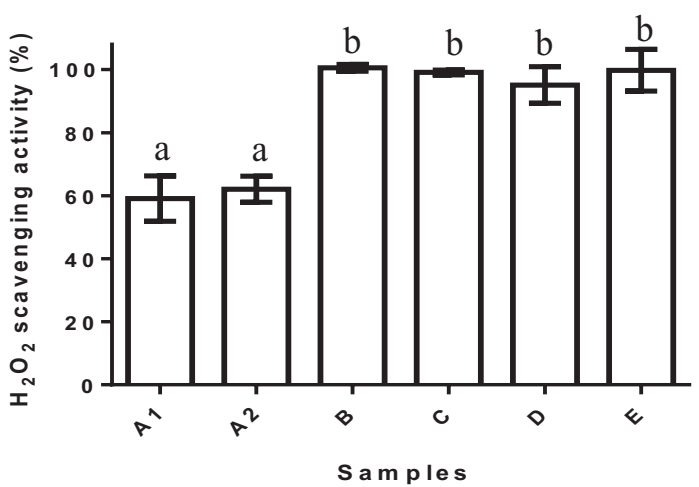

C

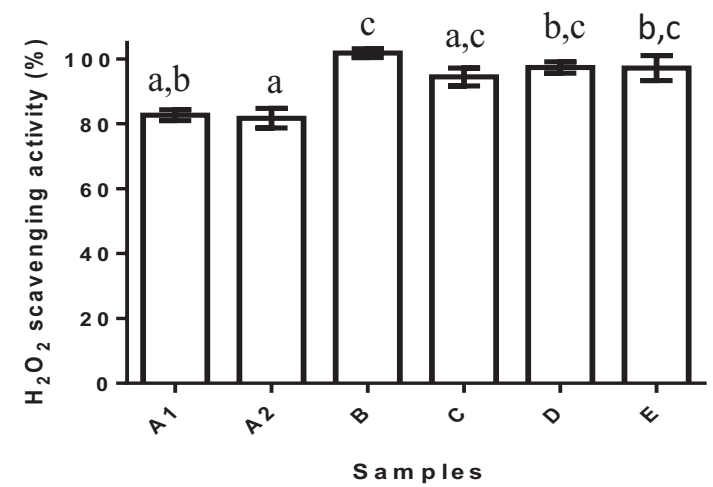

Fig. 6. $\mathrm{H}_{2} \mathrm{O}_{2}$ scavenging activity of the aqueous extract (AE) from A. muricata and of the nanoformulations at $30 \mu \mathrm{g} / \mathrm{ml}$ (A), $60 \mu \mathrm{g} / \mathrm{ml}$ (B) and $100 \mu \mathrm{g} / \mathrm{ml}$ (C). Data are presented as mean \pm SEM. A1 - treated AE; A2 - AE; B- phytosomes functionalized with $\mathrm{mApoE}$ (mApoE-P-AE); C- phytosomes with cholesterol and functionalized with $\mathrm{mApoE}$ (mApoE-PC-AE); D - liposomes functionalized with $\mathrm{mApoE}$ (mApoE-L-AE); E - liposomes with cholesterol and functionalized with mApoE (mApoE-LC-AE). Different letters mean significant differences at $p<.05$.

\section{Scavenging activity against hydrogen peroxide}

A significant increase in oxidative and inflammatory stress biomarkers has been described in MDD patients, supporting the oxidative stress etiological hypothesis. MAO enzymatic activity over the monoamines is a source of $\mathrm{H}_{2} \mathrm{O}_{2}$, which may directly disturb the brain redox imbalance or further produce highly reactive hydroxyl radicals via Fenton reaction (Stafford et al., 2007; Liu et al., 2008; Zhou et al., 2014a).

The AE displayed a strong scavenging activity against $\mathrm{H}_{2} \mathrm{O}_{2}$. The calculated IC $_{50}$ value of $48.9 \pm 2.5 \mu \mathrm{g} / \mathrm{ml}$ obtained for the $A E$ is 
comparable to that of the reference $\mathrm{H}_{2} \mathrm{O}_{2}$ scavenger ascorbic acid $(51.2 \pm 4.6 \mu \mathrm{g} / \mathrm{ml})$, under the same experimental conditions. At the three tested concentrations (30, 60 and $100 \mu \mathrm{g} / \mathrm{ml}$ ), mApoE-L-AE, mApoE-LC-AE, mApoE-P-AE and mApoE-PC-AE were more active than the parent extract (Fig. 6). This behavior is in accordance with the results reported by Landi-Librandi et al., (2012) who observed that liposomal flavonoids encapsulated in soya phosphatidylcholine:cholesterol or in soya phosphatidylcholine: cholesteryl ethyl ether kept their scavenging activity against $\mathrm{H}_{2} \mathrm{O}_{2}$. A low ratio between phospholipid and cholesterol or cholesteryl ethyl ether also favors the antioxidant activity.

\section{Conclusions}

The $\mathrm{AE}$ from $A$. muricata leaves revealed a phenolic-rich composition potentially exploitable for its neuroprotective effects. HPLC-DADESI-MS $^{n}$ analyses of the AE also allow the detection of trace amounts of the alkaloids reticuline and coclaurine but no acetogenins were detected, pointing to the safety of its consumption. This work demonstrated that phospholipid nanoformulations functionalized with $\mathrm{mApoE}$ are interesting candidates to deliver antioxidants and MAO-A inhibitors into the brain. Taking into consideration that the bioavailability of these compounds can be enhanced, it is expected that they will interact in a larger scale with multiple targets and pathways. The in vitro experimental limitations inherent to the vehicles' characteristics may be irrelevant in future in vivo assays, since the nanoformulations will release the loaded extract.

\section{Conflict of interest}

The authors declare that there are no conflicts of interest.

\section{Acknowledgments}

This work was financed through project UID/QUI/50006/2013, receiving financial support from FCT/MEC through national funds, and co-financed by FEDER, under the Partnership Agreement PT2020. C. G. thanks FCT for the FCT Investigator(/IF/01332/2014/CP1255/ CT0001). The authors would also like to acknowledge Sílvia Maia from CEMUP for her technical assistance with LTQ Orbitrap XL analyses and to Professor Michael Clifford for his advices in analyzing the MS spectra of phenolic acids.

\section{References}

Ajazuddin, Saraf, S., 2010. Applications of novel drug delivery system for herbal formulations. Fitoterapia 81 (7), 680-689.

Alali, F.Q., Liu, X.-X., McLaughlin, J.L., 1999. Annonaceous Acetogenins: recent progress. J. Nat. Prod. 62 (3), 504-540.

Amaral, J.S., Ferreres, F., Andrade, P.B., Valentão, P., Pinheiro, C., Santos, A., Seabra, R., 2005. Phenolic profile of hazelnut (Corylus Avellana L.) leaves cultivars grown in Portugal. Nat. Prod. Res. 19 (2), 157-163.

Balducci, C., Mancini, S., Minniti, S., La Vitola, P., Zotti, M., Sancini, G., Mauri, M., Cagnotto, A., Colombo, L., Fiordaliso, F., Grigoli, E., Salmona, M., Snellman, A., Haaparanta-Solin, M., Forloni, G., Masserini, M., Re, F., 2014. Multifunctional liposomes reduce brain $\beta$-amyloid burden and ameliorate memory impairment in Alzheimer's disease mouse models. J. Neurosci. 34 (42), 14022-14031.

Bana, L., Minniti, S., Salvati, E., Sesana, S., Zambelli, V., Cagnotto, A., Orlando, A. Cazzaniga, E., Zwart, R., Scheper, W., Masserini, M., Re, F., 2014. Liposomes bi- functionalized with phosphatidic acid and an ApoE-derived peptide affect A $\beta$ aggregation features and cross the blood-brain-barrier: implications for therapy of Alzheimer disease. Nanomedicine 10 (7), 1583-1590.

Bernardo, J., Ferreres, F., Gil-Izquierdo, Á., Valentão, P., Andrade, P.B., 2017. Medicinal species as MTDLs: Turnera diffusa Willd. Ex Schult inhibits CNS enzymes and delays glutamate excitotoxicity in SH-SY5Y cells via oxidative damage. Food Chem. Toxicol. 106 (Part A), 466-476.

Bicker, J., Alves, G., Fortuna, A., Falcão, A., 2014. Blood-brain barrier models and their relevance for a successful development of CNS drug delivery systems: a review. Eur. J. Pharm. Biopharm. 87 (3), 409-432.

Bikomo, E.O., Ebuehi, O.A.T., Magbagbeola, O.A., 2017. Antidepressant activity of ethanol leaf extract of Annona muricata L., in Sprague-Dawley rats. Am. J. Biochem. Biotechnol. 7, 1-5.

Briuglia, M.-L., Rotella, C., McFarlane, A., Lamprou, D.A., 2015. Influence of cholesterol on liposome stability and on in vitro drug release. Drug Deliv. Transl. Res. 5 (3), 231-242.

Caparros-Lefebvre, D., Elbaz, A., 1999. Possible relation of atypical parkinsonism in the French West Indies with consumption of tropical plants: a case-control study. Lancet 354 (9175), 281-286.

Carvalho, S., Santos, M., Lima, L., Mota-Pereira, J., Pimentel, P., Maia, D., Correia, D., Gomes, S., Cruz, A., Medeiros, R., 2017. IL6-174 G > C genetic polymorphism influences antidepressant treatment outcome. Nord. J. Psychiatry 71 (2), 158-162.

Cecchelli, R., Dehouck, B., Descamps, L., Fenart, L., Buée-Scherrer, V., Duhem, C. Lundquist, S., Rentfel, M., Torpier, G., Dehouck, M.P., 1999. In vitro model for evaluating drug transport across the blood-brain barrier. Adv. Drug Deliv. Rev. 36 (2), 165-178.

Champy, P., Melot, A., Guérineau Eng, V., Gleye, C., Fall, D., Höglinger, G.U., Ruberg, M., Lannuzel, A., Laprévote, O., Laurens, A., Hocquemiller, R., 2005. Quantification of acetogenins in Annona muricata linked to atypical parkinsonism in Guadeloupe. Mov. Disord. 20 (12), 1629-1633.

Clifford, M.N., Johnston, K.L., Knight, S., Kuhnert, N., 2003. Hierarchical scheme for LC $\mathrm{MS}^{\mathrm{n}}$ identification of chlorogenic acids. J. Agric. Food Chem. 51 (10), 2900-2911.

Cuyckens, F., Rozenberg, R., Hoffmann, E., Claeys, M., 2001. Structure characterization of flavonoid $O$-diglycosides by positive and negative nano-electrospray ionization ion trap mass spectrometry. J. Mass Spectrom. 36 (11), 1203-1210.

Dittmann, K., Riese, U., Hamburger, M., 2004. HPLC-based bioactivity profiling of plant extracts: a kinetic assay for the identification of monoamine oxidase-A inhibitors using human recombinant monoamine oxidase-A. Phytochemistry 65 (21), 2885-2891.

Ferraz, C.R., Silva, D.B., Prado, L.C.d.S., Canabrava, H.A.N., Bispo-da-Silva, L.B., 2017. Antidiarrhoeic effect and dereplication of the aqueous extract of Annona crassiflora (Annonaceae). Nat. Prod. Res 8, 1-5.

Ferreres, F., Pereira, D.M., Valentão, P., Andrade, P.B., Seabra, R.M., Sottomayor, M., 2008. New phenolic compounds and antioxidant potential of Catharanthus roseus. J Agric Food Chem 56 (21), 9967-9974.

Ferreres, F., Vinholes, J., Gil-Izquierdo, A., Valentão, P., Gonçalves, R.F., Andrade, P.B., 2013. In vitro studies of $\alpha$-glucosidase inhibitors and antiradical constituents of Glandora diffusa (Lag.) D.C. Thomas infusion. Food Chem. 136 (3), 1390-1398.

Florence, N.T., Benoit, M.Z., Jonas, K., Alexandra, T., Désiré, D.D.P., Pierre, K., Théophile, D., 2014. Antidiabetic and antioxidant effects of Annona muricata (Annonaceae), aqueous extract on streptozotocin-induced diabetic rats. J. Ethnopharmacol. 151 (2), 784-790.

George, V.C., Kumar, D.R.N., Suresh, P.K., Kumar, R.A., 2015. Antioxidant, DNA protective efficacy and HPLC analysis of Annona muricata (soursop) extracts. J. Food Sci. Technol. 52 (4), 2328-2335.

Gobbi, M., Re, F., Canovi, M., Beeg, M., Gregori, M., Sesana, S., Sonnino, S., Brogioli, D., Musicanti, C., Gasco, P., Salmona, M., Masserini, M.E., 2010. Lipid-based nanoparticles with high binding affinity for amyloid- $\beta 1-42$ peptide. Biomaterials 31 (25), 6519-6529.

Gregori, M., Bertani, D., Cazzaniga, E., Orlando, A., Mauri, M., Bianchi, A., Re, F., Sesana, S., Minniti, S., Francolini, M., Cagnotto, A., Salmona, M., Nardo, L., Salerno, D., Mantegazza, F., Masserini, M., Simonutti, R., 2015. Investigation of functionalized poly(N,N-dimethylacrylamide)-block-polystyrene nanoparticles as novel drug delivery system to overcome the blood-brain barrier in vitro. Macromol. Biosci. 15 (12), 1687-1697.

Grosso, C., Jäger, A.K., Staerk, D., 2013. Coupling of a high-resolution monoamine oxidase-A inhibitor assay and HPLC-SPE-NMR for advanced bioactivity profiling of plant extracts. Phytochem. Anal. 24 (2), 141-147.

Grosso, C., Valentão, P., Andrade, C., Andrade, P.B., 2015. HPLC-DAD analysis and in vitro enzyme inhibition: an integrated approach to predict herbal binary mixture behaviour employing median effect equation. Microchem. J. 119 (Supplement C), 176-182.

Gülçin, I., Mshvildadze, V., Gepdiremen, A., Elias, R., 2006. Screening of antiradical and antioxidant activity of monodesmosides and crude extract from Leontice smirnowii tuber. Phytomedicine 13 (5), 343-351.

Holt, A., Sharman, D.F., Baker, G.B., Palcic, M.M., 1997. A continuous spectrophotometric assay for monoamine oxidase and related enzymes in tissue homogenates. Anal. Biochem. 244 (2), 384-392.

Huang, R.H., Faulkner, R., 1981. The role of phospholipid in the multiple functional forms of brain monoamine oxidase. J. Biol. Chem. 256 (17), 9211-9215.

Justino, A.B., Pereira, M.N., Peixoto, L.G., Vilela, D.D., Caixeta, D.C., de Souza, A.V., Teixeira, R.R., Silva, H.C.G., de Moura, F.B.R., Moraes, I.B., Espindola, F.S., 2017 Hepatoprotective properties of a polyphenol-enriched fraction from Annona crassiflora Mart. fruit peel against diabetes-induced oxidative and nitrosative stress. J. Agric. Food Chem. 65 (22), 4428-4438.

Lage, G.A., Medeiros, F.d.S., Furtado, W.d.L., Takahashi, J.A., Filho, J.D.d.S., Pimenta 
L.P.S., 2014. The first report on flavonoid isolation from Annona crassiflora Mart. Nat. Prod. Res. 28 (11), 808-811.

Landi-Librandi, A.P., Caleiro Seixas Azzolini, A.E., de Oliveira, C.A., Lucisano-Valim, Y.M., 2012. Inhibitory activity of liposomal flavonoids during oxidative metabolism of human neutrophils upon stimulation with immune complexes and phorbol ester. Drug Deliv. 19 (4), 177-187.

Lannuzel, A., Höglinger, G.U., Verhaeghe, S., Gire, L., Belson, S., Escobar-Khondiker, M., Poullain, P., Oertel, W.H., Hirsch, E.C., Dubois, B., Ruberg, M., 2007. Atypical parkinsonism in Guadeloupe: a common risk factor for two closely related phenotypes? Brain 130 (3), 816-827.

Le Ven, J., Schmitz-Afonso, I., Lewin, G., Brunelle, A., Touboul, D., Champy, P., 2014. Identification of the environmental neurotoxins annonaceous acetogenins in an Annona cherimolia Mill. alcoholic beverage using HPLC-ESI-LTQ-Orbitrap. J. Agric. Food Chem. 62 (34), 8696-8704.

Leboeuf, M., Legueut, C., Cavé, A., Desconclois, J.F., Forgacs, P., Jacquemin, H., 1981. Alcaloïdes des annonacées XXIX: alcaloïdes de l'Annona muricata L. Planta Med 42 (05), 37-44.

Lin, R.D., Hou, W.C., Yen, K.Y., Lee, M.H., 2003. Inhibition of monoamine oxidase B (MAO-B) by Chinese herbal medicines. Phytomedicine 10 (8), 650-656.

Liu, J.-R., Chen, G.-F., Shih, H.-N., Kuo, P.-C., 2008. Enhanced antioxidant bioactivity of Salvia miltiorrhiza (Danshen) products prepared using nanotechnology. Phytomedicine 15 (1), 23-30.

Mancini, S., Minniti, S., Gregori, M., Sancini, G., Cagnotto, A., Couraud, P.-O., OrdóñezGutiérrez, L., Wandosell, F., Salmona, M., Re, F., 2016. The hunt for brain A $\beta$ oligomers by peripherally circulating multi-functional nanoparticles: potential therapeutic approach for Alzheimer disease. Nanomedicine 12 (1), 43-52.

Marques, V., Farah, A., 2009. Chlorogenic acids and related compounds in medicinal plants and infusions. Food Chem 113 (4), 1370-1376.

Masserini, M., 2013. Nanoparticles for brain drug delivery. ISRN Biochem. 2013 Article ID 238428, 18 pages.

Masserini, M., Re, F., Sancini, G., Forloni, G., Salmona, M. (2014). Liposomes active in vivo on neurodegenerative diseases, patent US 8877236 B2.

Matsushige, A., Kotake, Y., Matsunami, K., Otsuka, H., Ohta, S., Takeda, Y., 2012a Annonamine, a new aporphine alkaloid from the leaves of Annona muricata. Chem. Pharm. Bull. 60 (2), 257-259.

Matsushige, A., Matsunami, K., Kotake, Y., Otsuka, H., Ohta, S., 2012b. Three new megastigmanes from the leaves of Annona muricata. J. Nat. Med. 66 (2), 284-291.

Moreira, M.M., Barroso, M.F., Boeykens, A., Withouck, H., Morais, S., Delerue-Matos, C., 2017. Valorization of apple tree wood residues by polyphenols extraction: comparison between conventional and microwave-assisted extraction. Ind. Crops Prod. 104 (Supplement C), 210-220.

N'Gouemo, P., Koudogbo, B., Tchivounda, H.P., Akono-Nguema, C., Etoua, M.M., 1997 Effects of ethanol extract of Annona muricata on pentylenetetrazol-induced convulsive seizures in mice. Phytother. Res. 11 (3), 243-245.

NałẸcz, M.J., Zborowski, J., Famulski, K.S., Wojtczak, L., 1980. Effect of phospholipid composition on the surface potential of liposomes and the activity of enzymes incorporated into liposomes. Eur. J. Biochem. 112 (1), 75-80.

Navarro-Welch, C., McCauley, R.B., 1982. An evaluation of phospholipids as regulators of monoamine oxidase A and monoamine oxidase B activities. J. Biol. Chem. 257 (22), 13645-13649.

Nawwar, M., Ayoub, N., Hussein, S., Hashim, A., El-Sharawy, R., Wende, K., Harms, M., Lindequist, U., 2012. Flavonol triglycoside and investigation of the antioxidant and cell stimulating activities of Annona muricata Linn. Arch. Pharm. Res. 35 (5) 761-767.

Neves, A.R., Queiroz, J.F., Reis, S., 2016. Brain-targeted delivery of resveratrol using solid lipid nanoparticles functionalized with apolipoprotein E. J. Nanobiotechnology 14 (1), 27

Novaroli, L., Reist, M., Favre, E., Carotti, A., Catto, M., Carrupt, P.-A., 2005. Human recombinant monoamine oxidase $B$ as reliable and efficient enzyme source for inhibitor screening. Bioorganic Med. Chem. 13 (22), 6212-6217.
Omidi, Y., Campbell, L., Barar, J., Connell, D., Akhtar, S., Gumbleton, M., 2003 Evaluation of the immortalised mouse brain capillary endothelial cell line, b.End3, as an in vitro blood-brain barrier model for drug uptake and transport studies. Brain Res. 990 (1), 95-112.

Orlando, A., Re, F., Sesana, S., Rivolta, I., Panariti, A., Brambilla, D., Nicolas, J., Couvreur, P., Andrieux, K., Masserini, M., Cazzaniga, E., 2013. Effect of nanoparticles binding $\beta$-amyloid peptide on nitric oxide production by cultured endothelial cells and macrophages. Int. J. Nanomed. 8, 1335-1347.

Re, F., Cambianica, I., Zona, C., Sesana, S., Gregori, M., Rigolio, R., La Ferla, B., Nicotra, F., Forloni, G., Cagnotto, A., Salmona, M., Masserini, M., Sancini, G., 2011. Functionalization of liposomes with ApoE-derived peptides at different density affects cellular uptake and drug transport across a blood-brain barrier model. Nanomedicine 7 (5), 551-559.

Reavill, C.A., Wooster, M.S., Plummer, D.T., 1978. The interaction of purified acetylcholinesterase from pig brain with liposomes. Biochem. J. 173 (3), 851-856.

Rockenbach, I.I., Jungfer, E., Ritter, C., Santiago-Schübel, B., Thiele, B., Fett, R., Galensa, R., 2012. Characterization of flavan-3-ols in seeds of grape pomace by CE, HPLCDAD-MS $^{\mathrm{n}}$ and LC-ESI-FTICR-MS. Food Res. Int. 48 (2), 848-855.

Rottscholl, R., Haegele, M., Jainsch, B., Xu, H., Respondek, G., Höllerhage, M., Rösler, T.W., Bony, E., Le Ven, J., Guérineau, V., Schmitz-Afonso, I., Champy, P., Oertel, W.H., Yamada, E.S., Höglinger, G.U., 2016. Chronic consumption of Annona muricata juice triggers and aggravates cerebral tau phosphorylation in wild-type and MAPT transgenic mice. J. Neurochem. 139 (4), 624-639.

Santos, S.A.O., Vilela, C., Camacho, J.F., Cordeiro, N., Gouveia, M., Freire, C.S.R., Silvestre, A.J.D., 2016. Profiling of lipophilic and phenolic phytochemicals of four cultivars from cherimoya (Annona cherimola Mill.). Food Chem 211, 845-852.

Schmidt, J., Raith, K., Boettcher, C., Zenk, M.H., 2005. Analysis of benzylisoquinolinetype alkaloids by electrospray tandem mass spectrometry and atmospheric pressure photoionization. Eur. J. Mass Spectrom. 11 (3), 325-333.

Schmidt, K., Li, Z., Schubert, B., Huang, B., Stoyanova, S., Hamburger, M., 2003. Screening of entomopathogenic Deuteromycetes for activities on targets involved in degenerative diseases of the central nervous system. J. Ethnopharmacol. 89 (2), 251-260.

Stafford, G.I., Pedersen, P.D., Jäger, A.K., Van Staden, J., 2007. Monoamine oxidase inhibition by southern African traditional medicinal plants. S. Afr. J. Bot. 73 (3), 384-390.

Stévigny, C., Jiwan, J.-L.H., Rozenberg, R., de Hoffmann, E., Quetin-Leclercq, J., 2004. Key fragmentation patterns of aporphine alkaloids by electrospray ionization with multistage mass spectrometry. Rapid Commun. Mass Spectrom. 18 (5), 523-528.

Stewart, J.C.M., 1980. Colorimetric determination of phospholipids with ammonium ferrothiocyanate. Anal. Biochem. 104 (1), 10-14.

Weissbach, H., Smith, T.E., Daly, J.W., Witkop, B., Udenfriend, S., 1960. A rapid spectrophotometric assay of monoamine oxidase based on the rate of disappearance of kynuramine. J. Biol. Chem. 235 (4), 1160-1163.

Yan, L., Hu, Q., Mak, M.S., Lou, J., Xu, S.L., Bi, C.W., Zhu, Y., Wang, H., Dong, T.T., Tsim, K.W., 2016. A Chinese herbal decoction, reformulated from Kai-Xin-San, relieves the depression-like symptoms in stressed rats and induces neurogenesis in cultured neurons. Sci Rep. 6, 30014

Yu, F., Li, Y., Chen, Q., He, Y., Wang, H., Yang, L., Guo, S., Meng, Z., Cui, J., Xue, M., Chen, X.D., 2016. Monodisperse microparticles loaded with the self-assembled berberine-phospholipid complex-based phytosomes for improving oral bioavailability and enhancing hypoglycemic efficiency. Eur. J. Pharm. Biopharm. 103 (Supplement C), 136-148

Zhou, N., Tang, Y., Keep, R.F., Ma, X., Xiang, J., 2014a. Antioxidative effects of Panax notoginseng saponins in brain cells. Phytomedicine 21 (10), 1189-1195.

Zhou, Y., Li, H.-q., Lu, L., Fu, D.-1., Liu, A.-j., Li, J.-h., Zheng, G.-q., 2014b. Ginsenoside Rg1 provides neuroprotection against blood brain barrier disruption and neurological injury in a rat model of cerebral ischemia/reperfusion through downregulation of aquaporin 4 expression. Phytomedicine 21 (7), 998-1003. 